\title{
Broad Absorption Line Variability in Repeat Quasar Observations from the Sloan Digital Sky Survey
}

\author{
Britt F. Lundgren ${ }^{1}$, Brian C. Wilhite ${ }^{1,2}$, Robert J. Brunner ${ }^{1,2}$, Patrick B. Hall ${ }^{3}$, Donald P. \\ Schneider $^{4}$, Donald G. York ${ }^{5,6}$, Daniel E. Vanden Berk ${ }^{4}$, and Jonathan Brinkmann ${ }^{7}$
}

\begin{abstract}
We present a time-variability analysis of 29 broad absorption line quasars (BALQSOs) observed in two epochs by the Sloan Digital Sky Survey (SDSS). These spectra are selected from a larger sample of BALQSOs with multiple observations by virtue of exhibiting a broad CIV $\lambda 1549$ absorption trough separated from the rest frame of the associated emission peak by more than 3600 $\mathrm{km} \mathrm{s}^{-1}$. Detached troughs facilitate higher precision variability measurements, since the measurement of the absorption in these objects is not complicated by variation in the emission line flux. We have undertaken a statistical analysis of these detached-trough BALQSO spectra to explore the relationships between BAL features that are seen to vary and the dynamics of emission from the quasar central engine. We have measured variability within our sample, which includes three strongly variable BALs. We have also verified that the statistical behavior of the overall sample agrees with current model predictions and previous studies of BAL variability. Specifically, we observe that the strongest BAL variability occurs among the smallest equivalent width features and at velocities exceeding $12,000 \mathrm{~km} \mathrm{~s}^{-1}$, as predicted by recent disk-wind modeling.
\end{abstract}

Subject headings: quasars: general

\footnotetext{
${ }^{1}$ Department of Astronomy, University of Illinois at Urbana-Champaign 1002 W. Green St. Urbana, IL 61801

${ }^{2}$ National Center for Supercomputing Applications, Champaign, IL 61820

${ }^{3}$ Department of Physics and Astronomy, York University, 4700 Keele St., Toronto, ON, M3J1P3, Canada

${ }^{4}$ Department of Astronomy and Astrophysics, The Pennsylvania State University, 525 Davey Laboratory, University Park, PA 16802, USA

${ }^{5}$ Department of Astronomy and Astrophysics, University of Chicago, Chicago, IL 60637, USA

${ }^{6}$ Enrico Fermi Institute, University of Chicago, Chicago, IL 60637, USA

${ }^{7}$ Apache Point Observatory, 2001 Apache Point Road, PO Box 59, Sunspot, NM 88349, USA
} 


\section{Introduction}

Broad absorption lines (BALs) in quasar spectra are thought to be caused by obscuring overdensities in a wind, which is likely produced near the central accretion disk and propogated radially at some angle above an optically thick torus (e.g., Murray et al. 1995; Elvis 2000; Proga et al. 2000). BALs, which by definition exhibit a full width at half maximum (FHWM) of more than 2,000 $\mathrm{km} \mathrm{s}^{-1}$, have been observed at velocities as great as 66,000 $\mathrm{km} \mathrm{s}^{-1}$ in the quasar rest frame (Foltz et al. 1983). Mini-BALs compose a similar class of somewhat narrower lines, also considered intrinsic to the quasar, which are characterized by a FWHM in the range of 300-2,000 $\mathrm{km} \mathrm{s}^{-1}$ (Hamann \& Sabra 2004). In general, BALs are observed in 15-20\% of quasars (Weymann et al. 1991; Reichard et al. 2003). This percentage is typically interpreted as the fraction of the viewing angle obscured by these overdensities, although the occurence of BALs may instead indicate a particular phase of quasar evolution (Green et al. 2001; Yuan \& Wills 2003).

The accretion process which fuels the central engine of a quasar is prone to dramatic activity, presumably due to both internal turbulence in the surrounding accretion disk and the strong gravitational effects of a supermassive black hole. As a result, stochastic variations in the radiative output of quasars have been observed (e.g., Vanden Berk et al. 2004; Wilhite et al. 2005), and such variability can be expected to propagate into the observational properties of any matter confined within the disk wind (Murray et al. 1995; Elvis 2000; Proga et al. 2000). Variability of the equivalent widths of intrinsic absorption lines such as BALs may indicate a changing level of ionization in regions very near to the quasar central engine or a change in the covering factor or optical depth of the absorbing gas. Changes in the velocity separation between absorption features and corresponding emission lines can provide valuable insight into the wind acceleration mechanism, which in turn can help to illuminate the overall structure and physical properties of the quasar.

Data from large surveys such as the Sloan Digital Sky Survey (SDSS; York et al. 2000) have significantly increased the number of known BALQSOs in the past decade. While large catalogs of BALQSOs now exist (Reichard et al. 2003; Trump et al. 2006), to date, minimal work has been done with regard to their variability. Narrow intrinsic absorption line variability has already been detected in a number of studies (e.g., Hamann et al. 1995, 1997; Ganguly et al. 2001; Wise et al. 2004; Misawa et al. 2005), and BAL variability has been previously observed in a few objects (Foltz et al. 1987; Turnshek et al. 1988; Smith \& Penston 1988; Barlow et al. 1992; Vilkoviskij \& Irwin 2001; Ma 2002). Some studies have reported equivalent width variability in $\sim 25-33 \%$ of these broad features (Barlow 1994; Naravanan et al. 2004), yet no large statistical variability study has been published. The time-variability analysis by Barlow (1994), which examines 23 BALQSOs, is the largest 
study of this kind to date. Repeat spectroscopic observations from the SDSS provide a unique opportunity to study the variation in these sources, since the sample size is large and the homogeneity of SDSS data allows for precise measurements of line variability.

In this paper, we analyze a sample of 29 SDSS quasars with two-epoch observations and prominant CIV $\lambda 1549$ BALs that are effectively detached from the associated emission peak. Though considerably smaller than the latest BAL quasar samples, this work is the largest comprehensive spectroscopic variability study of BALQSOs to date.

\section{Observations and Data Reduction}

\subsection{The Sloan Digital Sky Survey}

Through June 2005, the SDSS had imaged $8000 \mathrm{deg}^{2}$ and obtained follow-up spectra for nearly $7 \times 10^{5}$ galaxies and $9 \times 10^{4}$ quasars. Imaging data are acquired by a 54-chip drift-scan camera (Gunn et al. 1998, 2006) on the dedicated 2.5-meter telescope at Apache Point Observatory in New Mexico. The data are reduced and calibrated by the PHOTO software pipeline (Lupton et al. 2001). The photometric system is normalized such that the SDSS $u, g, r, i$ and $z$ magnitudes (Fukugita et al. 1996) are on the AB system (Smith et al. 2002). A 0.5-meter telescope monitors site photometric quality and extinction (Hogg et al. 2001). Point source astrometry for the survey is accurate to less than 100 milliarcseconds (Pier et al. 2003), and imaging quality control is discussed in Ivezić et al. (2004).

A fraction of the objects located in the imaging are targeted for follow-up spectroscopy as candidate galaxies (Strauss et al. 2002; Eisenstein et al. 2001), quasars (Richards et al. $2002 \mathrm{~b}$ ), or stars (Stoughton et al. 2002). Targeted objects are grouped in 3-degree diameter tiles (Blanton et al. 2003) and aluminum plates are drilled with 640 holes at positions corresponding to the objects' sky locations. When the telescope is in spectroscopic mode, plates are placed in the imaging plane of the telescope and plugged with optical fibers that run from the telescope to twin spectrographs. Roughly 500 galaxies, 50 quasars and 50 stars are observed on each plate, and the remaining fibers are utilized for sky subtraction and calibration.

SDSS spectra cover the observer-frame optical and near infrared, from $3900 \AA-9100 \AA$, with a resolution of $\frac{\lambda}{\Delta \lambda} \approx 2000$ at $5000 \AA$ (Stoughton et al. 2002). Spectra are obtained in three or four consecutive 15-minute observations until an average minimum signal-to-noise ratio is met. Observations of 32 sky fibers, 8 reddening standard stars, and 8 spectrophotometric standard stars are used to calibrate the science targets' spectra. The Spectro2d pipeline flat-fields and flux calibrates the spectra and the Spectro1d code identifies spectral 
features and classifies objects by spectral type (Stoughton et al. 2002). Ninety-four percent of all SDSS quasars are identified spectroscopically by this automated calibration; the remaining quasars are identified through manual inspection. Quasars are defined to be those extragalactic objects with broad emissions lines (full width at half maximum velocity width $\gtrsim 1000 \mathrm{~km} \mathrm{~s},{ }^{-1}$ regardless of luminosity).

For this paper, we use spectra with additional calibration, as described in Wilhite et al. (2005). Through June 2004, objects corresponding to 181 plates had been observed multiple times, with time lags between observations ranging from days to years. These second observations, though originally unplanned, came as the product of normal survey operations, usually early in the survey, when a method had not yet been developed for determining when a plate had reached an adequate signal-to-noise-ratio. As discussed in Wilhite et al. (2005), spectra from plates observed greater than 50 days apart are guaranteed to have not been co-added and are more suitable for use in variability studies. There are 53 such large time-lag plate pairs containing almost 2200 quasars; single-epoch observations of 52 of these plate pairs are contained in the Fourth Data Release (DR4; Adelman-McCarthy et al. 2006).

\subsection{Refinement of Spectroscopic Calibration}

As was shown in Vanden Berk et al. (2004) and Wilhite et al. (2005), some additional spectrophotometric calibration of SDSS spectra is necessary for variability studies. The calibration methods used are summarized below; see Wilhite et al. (2005) for a complete discussion. The Spectro1d pipeline calculates three signal-to-noise ratios for each spectrum by calculating the median signal-to-noise ratio per pixel in the regions of the spectrum corresponding to the SDSS $g, r$ and $i$ filter transmission curves. Hereafter, when referring to the two halves of a plate pair we use the phrase "high-S/N epoch" to refer to the plate with the higher median $r$-band signal-to-noise ratio. The plate with the lower median $r$-band signal-to-noise ratio will be called the "low-S/N epoch." This is a plate-wide designation; although most objects follow the plate-wide trend, this does not speak to the relative $\mathrm{S} / \mathrm{N}$ values for any given individual object, nor does it correspond to an object's relative line or continuum flux at a given epoch. The stars on a plate are used to resolve calibration differences between the high- and low-S/N epochs, under the assumption that the majority of stars are non-variable (precautions are taken to remove the obviously variable stars from re-calibration, see Wilhite et al. (2005) for complete details). For each plate pair, we create a re-calibration spectrum, equal to the ratio of the median stellar high-S/N epoch flux to the median stellar low-S/N flux, as a function of wavelength. This re-calibration spectrum is fitted with a 5 th-order polynomial to preserve real wavelength dependences but remove 
pixel-to-pixel noise (c.f. Figure 5 of Wilhite et al. 2005), leaving a smooth, relatively featureless curve as a function of wavelength. All low-S/N epoch spectra are then scaled by this "correction" spectrum.

\subsection{BALQSO Sample Selection}

Each observation at both epochs of the 2200 objects compiled by Wilhite et al. (2005) was inspected by eye for the presence of CIV BAL and mini-BAL features. BALQSO spectra were selected for further study if they contained at least one CIV absorption feature with a velocity width of at least $1000 \mathrm{~km} \mathrm{~s}^{-1}$ in one or more epochs with no discernable doublet structure. Such doublets might indicate that the absorption is due to a collection of overlapping narrow CIV absorption lines rather than one broad absorber (Weymann et al. 1991).

Objects meeting these primary criteria were also tested to ensure that intervening absorption systems were not responsible for any of the apparent BALs, since MgII $\lambda 2800$ and FeII $\lambda(2261,2344,2374,2383,2587,2600)$ in multiple lower redshift absorption line systems have the potential to overlap and produce seemingly broad features in the region blueward of CIV. Such occurrences are easily recognized, as the MgII doublet exhibits a significantly

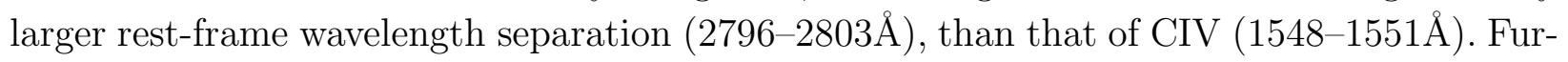
thermore, FeII absorption lines occur over a large wavelength range in the visible spectrum and are almost always accompanied by a strong MgII doublet with the same redshift. These characteristics ensure that even if low-ionization absorption lines are present in the wavelength range where intrinsic CIV absorption would be observed, these intervening absorbers may be accurately identified and removed from the CIV BALQSO sample.

Of the 1186 objects in this sample within the redshift range where the CIV emission line can be measured in the SDSS spectra $\left(1.6 \leq z_{q s o} \leq 4.2\right), 172$ (14.5\%) contain BALs that met the aforementioned criteria. This precentage of BALs within the quasar population agrees with the expected values from previous catalogs (see, e.g., Reichard et al. 2003; Hewett \& Foltz 2003).

Measurements of BAL variability are difficult to make, since in most cases the intrinsic nature of these lines produces an overlap with the quasar emission lines. This complication demands precise continuum and line profile fitting if one wishes to study the variability of absorption in the region of broad emission. It has also been established that the continua of

quasars are prone to much greater variability than the emission line flux (Wilhite et al. 2005). Therefore, any efforts to study the relationship between quasar source variability and changes 
in the intrinsic absorption features must cleanly separate the effects of variability in the quasar continuum and emission lines. By restricting our sample to those BALs which do not overlap with the broad CIV emission feature, we can obtain higher precision measurements of the equivalent widths of these features, and directly probe the correlation between quasar continuum emission and BAL variability.

As a result, we narrowed our initial sample of 172 multi-epoch SDSS BALQSOs to 29 that were found to contain broad absorption lines separated from the associated CIV emission line by at least $3600 \mathrm{~km} \mathrm{~s}^{-1}$ in the quasar rest frame. Vanden Berk et al. (2001) finds $1546.15 \AA$ to be the median wavelength of CIV emission in the composite spectrum of 2200 SDSS quasars and a line width $\left(\sigma_{\lambda}\right)$ of $14.33 \AA$. On the blue side of the emission peak, this width is approximately equivalent to a velocity of $3600 \mathrm{~km} \mathrm{~s}^{-1}$ with respect to CIV laboratory rest frame wavelength of $1549 \AA$. This lower-limit separation ensures that the absorption feature lies on a locally flat section of the spectrum which, at most, only marginally overlaps with the CIV emission region. Within these 29 spectra, we find 33 detached-trough BALs meeting the $3600 \mathrm{~km} \mathrm{~s}^{-1}$ criterion, as some quasars host more than one distinct BAL at high velocity. The observational details of the selected quasars are presented in Table 1.

\section{Data Analysis}

Although there has been some success in automating the identification of BALs from within a large sample of quasar spectra (Trump et al. 2006), the actual analysis of the BAL features within these spectra requires manual inspection. The inability to fully automate this process is largely due to the tendency of BAL widths and velocities in the quasar rest frame to vary greatly between objects. Furthermore, the continuum fitting, upon which any absorption profiles must be based, is complicated by the very presence of the BALs blueward of the emission peaks. For these reasons, each spectrum requires careful analysis to ensure that the wavelength ranges included in the continuum fitting and equivalent width measurements of the BAL features are appropriate. The rest of this section details the processes we used to perform measurements of the BAL features.

\subsection{Continuum Fitting}

To determine the appropriate continuum level we first de-redshifted each spectrum into the rest frame of the quasar. The SDSS pipeline occasionally calculates a different red- 
shift for the two epochs (e.g., due to signal-to-noise differences or incorrect emission line identifications). For consistency, we always use the SDSS redshift assigned to the higher signal-to-noise spectrum. Wilhite et al. (2006) showed that the shift in central wavelength of the CIV emission peak between epochs is negligible, even in variable quasars. Thus, a single high signal-to-noise calculation of the emission redshift should be satisfactory for both epochs. Since our goal is the measurement of variability in the absorption features, it is imperative that the redshift of the emitting source be consistent between observations, which ensures that all measurements incorporating velocity use the same standard scaling in both epochs.

After de-redshifting each spectrum into the rest-frame of the quasar, a continuum is fit across the region of CIV emission. Because the part of the spectrum analyzed here is relatively small (roughly $1400-1700 \AA$ in the rest frame of the quasar), the continuum is approximated by a linear fit in wavelength in the quasar's rest-frame, as was shown to be sufficient by Wilhite et al. (2006). For each object, two regions of the spectrum are included to construct this fit, one on either side of the CIV emission line.

In most cases, the part of the spectrum corresponding to the quasar rest frame wavelength range $1440-1460 \AA$ was used to fit the region blueward of intrinsic CIV features. However, in cases where this wavelength range contains a BAL, we chose a region at a longer wavelength that was still blueward of the CIV emission. As the location of the fit regions can vary from object to object, we present the wavelength boundaries used for the blue side of each fit in Table 2 .

Significant emission from other ions, such as FeII, is often found just redward of the CIV emission line (e.g., Wilkes 1984; Boyle 1990; Laor et al. 1994; Vanden Berk et al. 2001). As a result, the red side of the fit must be extended to longer wavelengths, where the spectrum returns to near-continuum levels (for a detailed discussion, see Wilhite et al. 2006). We have used the rest frame region $1680-1700 \AA$ to fit the red side of the continuum, as was done by Wilhite et al. (2006). Since 1680-1700A was always taken to be the range for the red component of these linear fits, this information has not been presented with the other fit parameters in Table 2.

Since BALs are thought to arise from absorption of gas at high velocities with respect to the emission line source, each spectrum (and each corresponding wavelength region to be fit) is translated into velocity space zeroed to the central CIV emission rest wavelength at $1549 \AA$. The velocity translation can thus be summarized with the formula:

$$
\frac{v}{c}=\left(\frac{R^{2}-1}{R^{2}+1}\right)
$$


where

$$
R=\left(\frac{1+z_{q s o}}{1+z_{a b s}}\right)
$$

$z_{a b s}=\left(\lambda_{a b s} / 1549 \AA\right)-1$ denotes the redshift of each part of an absorption feature, and $z_{q s o}$ is the SDSS high-S/N redshift of the quasar. The error on the absorber's redshift value is a function of the binning size and is estimated to be: $\sigma_{a b s}=\Delta \lambda_{a b s} /(1549 \AA)$. The error in the velocity measurement, $\sigma_{v}$, was then calculated with standard error propagation of $\sigma_{a b s}$ and the error on the calculated quasar redshift, $\sigma_{q s o}$, as determined by the SDSS.

A linear least squares fit in velocity space (in the quasar's rest-frame) of the continuum regions chosen for each spectrum was calculated using the POLYFIT routine in IDL. Table 2 also summarizes the fit parameters and their associated error, including the slopes $\left(m \pm \sigma_{m}\right)$ and the flux densities of each fit at $v=0 \mathrm{~km} \mathrm{~s}^{-1}\left(f_{c}(0) \pm \sigma_{f(0)}\right)$. We recover a linear fit to the continuum, $\left(f_{c}(v)=f_{c}(0)+m v\right)$, with the corresponding flux errors for each velocity bin:

$$
\sigma_{f_{c}(v)}=\sqrt{\sigma_{f(0)}^{2}+v^{2} \sigma_{m}^{2}+m^{2} c^{2} \sigma_{\beta}^{2}}
$$

\subsection{Equivalent Width}

Normalization provides the best method for removing the effect of continuum variability on the equivalent width measurements of the absorption features. With the spectra translated into velocity space and the continuum fitting complete, each flux-calibrated spectrum is therefore normalized by its respective linear continuum fit. The normalized flux density as a function of velocity in the quasar rest frame is given by:

$$
f_{n}(v)=\frac{f(v)}{f_{c}(v)}
$$

where $f(v)$ is the SDSS spectum with the additional flux calibration of Wilhite et al. (2005). The error on the flux density measurement in each velocity bin, $\sigma_{f(v)}$, is simply the respective SDSS error spectrum for each of the quasar observations (Stoughton et al. 2002) after the appropriate scaling as done in Wilhite et al. (2005). These error spectra are also normalized by dividing by continuum flux density (as determined by the fit) to preserve the percentage error in flux density in each bin.

The two-epoch normalized spectra and corresponding normalized SDSS errors are shown in Figure A1 of the Appendix. The upper and lower rest frame velocity boundaries of these BALs $\left(v_{\min }\right.$ and $v_{\max }$, respectively, shown as dotted vertical lines in Figure A1), between which the flux density does not rise to the level of the fitted continuum, were determined by 
visual inspection of each spectrum. A table of these BALs and their measured boundaries is presented in Table 3 .

Equivalent width measurements of the absorbers were calculated from the normalized spectra as follows:

$$
E W=\sum_{i=v_{\min }}^{v_{\max }}\left(1-f_{n}(i)\right) \Delta v_{i}
$$

An additional source of error must be considered when measuring the equivalent widths, since the original flux density has been divided by an approximated continuum fit. The error introduced by fitting propagates into the final equivalent width measurement of each BAL feature,

$$
\sigma_{E W}=\sqrt{\sum_{i=v_{\min }}^{v_{\max }}\left(\left(\frac{\sigma_{f(i)}}{f(i)}\right)^{2}+\left(\frac{\sigma_{f_{c}(i)}}{f_{c}(i)}\right)^{2}\right)\left(f_{n}(i)\right)^{2} \Delta v_{i}}
$$

where $\sigma_{f(i)} / f(i)$ is the fractional error of each non-normalized bin of flux, as determined from the SDSS error spectra, and $\Delta v_{i}$ is the velocity width of each pixel in $\mathrm{km} \mathrm{s}^{-1}$.

\subsection{BAL Variability}

To quantify variability in the equivalent widths of the BALs, we measure the fractional change, as given by: $\Delta E W /\langle E W\rangle$, where $\Delta E W=\left(E W_{2}-E W_{1}\right)$, and $\langle E W\rangle$ is taken to be the average value for the two epochs. Subscripts 1 and 2 refer to the first and second time epoch measurements respectively. It should be noted that for highly variable BALs these measurements of "fractional change" can appear deceptively small and do not reflect a percentage change. For instance, SDSS J081822.63+434633.8 exhibits a fractional change of 1.824 by this definition, yet its equivalent width has increased by more than a factor of 20 .

The absolute equivalent width variability of the 33 BALs in our sample is shown as a function of epoch separation time in the rest frame of the quasar in Figure 1. The positive correlation demonstrates that the most variable BALs have longer epoch separation times, as we should expect when observing real time-variability. If the variability distribution had been evenly spread throughout the range of epoch separation times, we might conclude that the observed variability was uncorrelated with time and could be representative of an artifact of the analysis. Instead, we see that the longer the time baseline between observations, the greater the potential for measuring variability, which is a general trend in quasar variability analyses. 
Figure 2 shows the absolute fractional change in BAL equivalent width as a function of average equivalent width. We see that the likelihood of detecting equivalent width variability decreases for absorbers with larger equivalent widths, an effect first noted by Barlow (1994). This behavior is what we might intuitively expect, since greater changes in continuum flux are required to ionize absorbers in larger troughs, due to the high optical depths and column densities of strong absorbers. However, continuum flux variation is not required to achieve variability in BALs, as a change in covering factor could produce similar changes in equivalent width and may be necessary to produce variability in the largest saturated features (Arav et al. 2001; Hall et al. 2002). Thus, a change in covering factor could explain the significant variability we observe in some large equivalent width BALs, while the overall trend favors variability in small equivalent width features.

We also analyze various BAL characteristics as a function of the overall BAL velocity, since absorption features observed at differing velocities are thought to probe geometrically disparate regions within the disk wind (see, e.g., Murrav et al. 1995; Proga et al. 2000). We calculate the BAL velocity by taking the difference between the unweighted average velocity of the absorber and the center of the CIV emission line at $v=0 \mathrm{~km} \mathrm{~s}^{-1}$ in the quasar rest frame. To investigate any correlation between the location of a BAL within the wind and its tendency to vary, we plot the absolute fractional change in BAL equivalent width as a function of BAL velocity in Figure 3. While a linear relationship between these two parameters is not obvious from these data, we do find that the most highly variable BALs in our sample are found at high velocities, namely, $12,000<\mathrm{v}<15,000 \mathrm{~km} \mathrm{~s}^{-1}$.

Having identified equivalent width variability within our sample, we proceed to parameterize the particular ways in which the equivalent widths are prone to variation. Since the changes in BAL equivalent widths can be triggered by bulk motion of the absorber along the line of sight, changes in the optical depth, covering factor, or the degree of ionization in the gas (see, e.g., Barlow et al. 1989; Hamann 1998; Narayanan et al. 2004), we seek to quantify the propensity for variability separately in these parameters. The effects of covering factor, ionization, and optical depth are complexly related, and efforts to separate these effects would require studying the variability of the CIV BAL in relation to broad absorption from other ions in these spectra. Since our study is limited exclusively to the CIV features, the separation of these parameters exceeds the scope of this paper. We instead explore variability in two parameters incorporated in the equivalent width measurement: the velocity width and the average residual flux density, hereafter referred to as the depth. Variability of the velocity width provides information about the bulk motion of the absorber, and monitoring changes in depth summarizes the effects of variation in ionization, covering factor, and optical depth. 
We define velocity width as the difference between velocity boundaries of the BAL, as determined by a visual inspection and measured in velocity units with respect to the quasar rest-frame emission. These widths have a lower limit of $1,000 \mathrm{~km} \mathrm{~s}^{-1}$, as required by our selection criteria, and effectively measure the velocity separation over which the flux density does not rise above the level of the continuum. These boundaries are given in Table 3. Due to the resolution of the spectra in the CIV region for an average redshift of $z \simeq 2(\sim 150$ $\mathrm{km} \mathrm{s}^{-1}$ ) and the uncertainty in defining the edges of the BAL troughs, we have assigned a conservative error of $300 \mathrm{~km} \mathrm{~s}^{-1}$ for each measurement of the boundary limits in velocity space. Velocity width measurements, therefore, have an associated error of $424 \mathrm{~km} \mathrm{~s}^{-1}$, and errors on measurements of the average BAL velocity are calculated similarly to be $212 \mathrm{~km}$ $\mathrm{s}^{-1}$.

It should be emphasized that the velocity distribution of our sample is limited by the range of velocities over which CIV BALs may be observed. The SiIV/OV broad emission line at $1400 \AA$ provides an observational upper limit for determining the CIV BAL velocities, which translates to a velocity of $\sim 28,000 \mathrm{~km} \mathrm{~s}^{-1}$ in the rest frame of the quasar. Additionally, our method of continuum fitting further restricts this maximum velocity, since the procedure we have implemented requires a relatively featureless continuum near $1460 \AA$, which corresponds to $\sim 19,000 \mathrm{~km} \mathrm{~s}^{-1}$ in the quasar rest frame. The minimum velocity of observed BALs in this sample is further restricted by our inclusion of detached-trough only features, which exhibit a separation from the emission line of at least $3600 \mathrm{~km} \mathrm{~s}^{-1}$. Thus, we can only select BALs over the velocity interval $3600-19,000 \mathrm{~km} \mathrm{~s}^{-1}$.

The depth of each BAL is recovered by simply dividing the calculated equivalent width of each feature by its measured velocity width at each epoch. This procedure results in a simple unweighted average normalized flux density for each BAL. The uncertainty of the measurement is determined by propagation of the errors assigned to the velocity width and the equivalent width.

As evidenced in Figures 1, 2, and 3, many of the BALs exhibit a fractional change in equivalent width that is consistent with zero. These objects may be removed for further BAL variability analysis, as they do not aid in characterizing the observed variability. Since one might expect to see variation in all BALs, given sufficiently long timescales, and since most of the BALs in our sample vary to some measurable degree, we can select a "significantly variable" subsample that is large enough to allow for statistical analysis but still limited to include only high precision data. The criteria for this selection was two-fold, allowing significant changes in equivalent width or velocity width to separately qualify a BAL as "significantly variable". BALs exhibiting either a fractional change in equivalent width with at least $2.5 \sigma$ significance or a fractional change in velocity width with at least $1 \sigma$ significance 
were included in this subsample. Fourteen BALs met the first criterion, while 7 met the latter. In all, 16 (half of the total BAL sample) were found to have significant variability by one or both criteria, indicating that significant changes in velocity width are generally, though not always, accompanied by equivalent width variability.

\subsection{Continuum Variability}

Previous studies have reported marginal evidence of a correspondence between changes in the continuum flux and BAL variability (Barlow et al. 1989; Barlow 1994). These results, however, note a high frequency of exceptions to this correlation, which implies that significant variability in a BAL can sometimes be seen in the absence of any change in the continuum flux. Presumably, this disparity results from a time delay between these variations, due to the distance separating the central source and the BAL region, for which lower limits of $(3 \mathrm{x}$ $\left.10^{17}\right)-\left(3 \times 10^{18}\right) \mathrm{cm}$ have been speculated (Murray et al. 1995). Given the timescales of our observations, such changes should be frequently out of phase and likely unobservable with only two epochs of observation. Thus, we do not expect to detect coincident variability of BALs with the quasar continuum in our two-epoch data. Additionally, changes in covering factor have been suggested as a common mechanism of BAL equivalent width variability, which is independent of variation in the continuum flux (Arav et al. 2001; Hall et al. 2002; Misawa et al. 2005).

To investigate the role of a changing continuum flux in our absorber variability analysis, we have quantified the continuum variability for each object. For our purposes, we define the continuum variability in each object as the fractional change in the flux density of the continuum fit evaluated at $1549 \AA\left(v=0 \mathrm{~km} \mathrm{~s}^{-1}\right)$. This velocity serves as a common reference point approximately midway between fitting regions, which has been chosen for consistency. We quantify the variability of the continuum as the fractional change in fitted continuum flux density at $v=0 \mathrm{~km} \mathrm{~s}^{-1}$ as: $\Delta f_{c}(0) /<f_{c}(0)>$, where $\Delta f_{c}(0)=\left(f_{c}(0)_{2}-f_{c}(0)_{1}\right)$, and $<f_{c}(0)>$ is the two-epoch averaged flux density of the fitted continuum, evaluated at the center of rest frame CIV emission $\left(v=0 \mathrm{~km} \mathrm{~s}^{-1}\right)$. Subscripts indicate the relevant epoch for each measurement.

Using this definition, the variability of the continuum for each object is shown as a function of epoch separation time in the quasar rest frame in Figure 4. A weighted least squares fit has been overplotted, which shows a convincing increase in variability with increasing time. This trend agrees with previous studies of continuum variability (see, e.g., Vanden Berk et al. 2004; Wilhite et al. 2006) while also revealing the inclusion in our sample of quasars with variable continua. In subsequent analyses, we uniquely identify quasars with 
significant continuum variability to highlight their differences. These "significantly variable" quasars, fifteen in number (half of the total quasar sample), were identified as such if they exhibited a fractional change in continuum flux that was more than four times the associated error.

\section{Discussion}

Having quantified significant variability among our sample of 33 BALs, we may further explore the ways in which this variability occurs. Figure 5 includes only the 16 significantly variable BALs selected by the aforementioned criteria (see §3.3). Here we track the changes in the BAL depths and widths with time. Arrows indicate the direction of change with time. In this figure, quasars hosting both significantly variable BALs and significantly variable continua (see §3.4) are marked at the first epoch measurement with diamonds. We see that the variable BAL sample draws a nearly equal representation from objects with both variable and non-variable continua, which gives an indication that our analysis has not been compromised by continuum variability.

The three most variable BALs are associated with variable continua, as we see in Figure 5, but among the larger sample, we do not see a strong correlation between continuum variability and BAL variability. In fact, two of the features exhibiting the greatest change in depth are associated with non-variable quasar continua. However, the overall propensity of these features to change in depth, rather than in width, suggests that changing levels of ionization in the outflow may indeed be the primary cause of the observed variability, as opposed to a bulk motion of the absorber (Barlow 1994). The argument for ionization-prompted variability is strengthened by the inverse correlation observed between the equivalent width and equivalent width variability among these BALs (as shown in Figure 2).

If we consider the primary mechanism of variability in these BALs to be changes in the ionizing flux from the central source, we can place limits on the electron densities of each BAL, as shown by Hamann et al. (1997); Narayanan et al. (2004); Misawa et al. (2005). We do so by taking a nominal gas temperature of 20,000K (Hamann et al. 1995) and assuming that the BALs are close to ionization equilibrium, with CIV as the dominant ionization state of $\mathrm{C}$. The rest-frame timescales of our observations may then be used to place upper limits on the recombination time for each absorber, and we may extract lower limits of the electron densities (see eq. [1] in Hamann et al. (1997); eq. [1] in Naravanan et al. (2004); discussion in Misawa et al. (2005)). We thereby calculate the limiting electron densities, $\mathrm{n}_{e} \geq 37,900$ $\mathrm{cm}^{-3}, \mathrm{n}_{e} \geq 51,000 \mathrm{~cm}^{-3}$, and $\mathrm{n}_{e} \geq 39,000 \mathrm{~cm}^{-3}$, respectively for the three most variable BALs: SDSS J075010.17+304032.3, SDSS J081822.63+434633.8, and SDSS J093620.52+004649.2. 
As seen in Figure 3, the three most highly variable BALs are observed in the velocity range 12,000-16,000 $\mathrm{km} \mathrm{s}^{-1}$. In Figure 6 we separate the parameters of depth and width to explore these factors of equivalent width variability separately as functions of velocity in the most significantly variable BALs. Observable changes in depth are much more common within the overall sample, as shown in Figure 5, and these changes occur with similar frequency over the entire velocity range of our sample. However, when velocity width variability occurs at a measurable level, it appears isolated within the same high velocity range where the most variable BALs are found. We also notice that the largest changes in velocity width are positive in sign, indicating that the material may be spreading due to changing levels of ionization, covering factor, or acceleration over a range of velocities.

This observation of strong BAL variability at large velocities agrees with the disk wind model of Proga et al. (2000), which predicts the production of variable overdensities in the fast streaming wind as a result of Kelvin-Hemholtz instabilities at $\sim 10,000 \mathrm{~km} \mathrm{~s}^{-1}$. Proga et al. (2000) also predict that such overdensities should reach a maximum velocity of $15,000 \mathrm{~km} \mathrm{~s}^{-1}$ in this stream, which is consistent with our observations. In Figure 5, we see that while many more of the troughs exhibit changes in depth, the most variable BALs all show a change in velocity width as well. This may indicate that, in some BALs, the degree of ionization or covering factor changes as a function of velocity, causing variability in the apparent breadth of the absorbing feature.

It should be emphasized that we have applied a conservative error of $212 \mathrm{~km} \mathrm{~s}^{-1}$ to each BAL velocity measurement (x-axis values in Figures 3, 5, and 6), as explained in §3.3. These errors are larger than most of the velocity displacements we observe between epochs, which is consistent with the fact that no previous studies of BAL time-variability have observed velocity changes greater than $125 \pm 63 \mathrm{~km} \mathrm{~s}^{-1}$ on timescales longer than 6 years in the quasar rest frame (Rupke et al. 2002). As the epoch separations of our data are significantly shorter and our spectral resolution much lower, we should not expect that any apparent changes in velocity in Figures 5 and 6 are significant.

\subsection{Interesting Objects}

Of the 33 BALs in our sample, three quasars demonstrated exceptionally strong equivalent width variability: SDSS J075010.17+304032.3 , SDSS J081822.63+434633.8, and SDSS J093620.52+004649.2. The observed absolute fractional changes in the equivalent widths of these BALs were $1.455 \pm 0.111,1.824 \pm 0.208$, and $1.499 \pm 0.234$, respectively. The amplitudes of variability seen in these three absorption features separates them from the other BALs in our sample by a substantial margin, since only one other BAL (SDSS J15026.66-003606.9) 
was found to exhibit an absolute fractional change greater than 0.70 (see Table 3).

The greatest measured variability within our sample is observed in SDSS J081822.63+434633.8 (Object 19 in Figure A1). The BAL present in this spectrum grows by a factor of 20 in equivalent width between observations. The trough of this BAL is asymmetric, with a noticably shallower slope on the red side. While the high velocity end of the BAL deepens between epochs, the velocity width of this feature nearly doubles, as the low velocity end of the trough extends to absorb more of the continuum flux in the later observation.

SDSS J075010.17+304032.3 (Object 28 in Figure A1) hosts the second-most variable BAL, which nearly disappears in the second epoch observation. The spectrum of this source is especially interesting, as it hosts at least two strong, narrow CIV absorption line systems, presumably also intrinsic to the quasar, that show little if any variability between epochs. The signal-to-noise of the second observation nearly matches that of the first, thus any variability displayed by this BAL is unlikely to be due to noise.

SDSS J093620.52+004649.2 (Object 17 in Figure A1) was found to be the third-most variable BAL. In contrast to SDSS J075010.17+304032.3, this BAL appears after being nearly undetectable in the first epoch. This BAL grows symmetrically between epochs, achieving both a greater depth of absorption and velocity width in the later observation.

\section{Conclusions}

We have detected significant time-variability among our sample of 36 detached-trough CIV BALs on time baselines shorter than a year in the quasar rest frame. Included in our sample are three exceptionally variable BALs that are observed in SDSS J075010.1+304032.3, SDSS J081822.63+434633.8, and SDSS J093620.52+004649.2. These three extremely variable BALs are observed within the velocity range $12,000-16,000 \mathrm{~km} \mathrm{~s}^{-1}$. The propensity for variability at these velocities is consistent with recent disk wind modeling by Proga et al. (2000), although other significantly variable BALs in our sample populate a much larger range in velocity $\left(6,000-16,000 \mathrm{~km} \mathrm{~s}^{-1}\right)$. Most of the variable BALs in our sample exhibit average equivalent widths of $\leq 2000 \mathrm{~km} \mathrm{~s}^{-1}$, and the three most strongly variable BALs represent some of the smallest features, with equivalent widths of $<1000 \mathrm{~km} \mathrm{~s}^{-1}$.

We find that the BALs primarily vary in depth on the timescales we observe, with fewer than half of the significantly variable troughs exhibiting a measurable change in velocity

width. This result is consistent with the findings of Barlow (1994) and may indicate that the dominant contribution to the BAL variability is a changing degree of ionization or covering 
factor. Since we find no strong correlation between changes in BAL equivalent width and continuum variability, it is likely that either the variability of the source is out of phase with the variability of the BAL region, or the changes in covering factor provide the primary mechanism for depth variability in these BALs.

The infrequency of observed changes in velocity likely reflects the very long timescales on which these features can be seen to accelerate. Future higher resolution spectra could improve measurements of velocity variability by enabling us to observe changes within the BAL structure and allowing for direct measurements of the acceleration within the wind. Furthermore, analyzing a similar sample of low-ionization BALs would allow us to explore the variability of BALs at greater outflow velocities, since quasar spectra are receptive to $\mathrm{MgII}$ and FeII absorption features over a larger portion of the visible spectrum. Future work with more epochs could also place limits on the timescales over which these strongly variable BALs might return to an equilibrium state, and additional monitoring of the three most highly variable BALs may be especially rewarding.

BFL, BCW, and RJB would like to acknowledge support from Microsoft Research, NASA through grants NAG5-12578 and NAG5-12580, and support through the NSF PACI Project. D.P.S. would like to acknowledge funding from NSF grant AST-0607634. P. B. H. is supported by NSERC. Additionally, we would like to thank the referee for many helpful comments and suggestions.

Funding for the SDSS and SDSS-II has been provided by the Alfred P. Sloan Foundation, the Participating Institutions, the National Science Foundation, the U.S. Department of Energy, the National Aeronautics and Space Administration, the Japanese Monbukagakusho, the Max Planck Society, and the Higher Education Funding Council for England. The SDSS Web Site is http://www.sdss.org/.

The SDSS is managed by the Astrophysical Research Consortium for the Participating Institutions. The Participating Institutions are the American Museum of Natural History, Astrophysical Institute Potsdam, University of Basel, Cambridge University, Case Western Reserve University, University of Chicago, Drexel University, Fermilab, the Institute for Advanced Study, the Japan Participation Group, Johns Hopkins University, the Joint Institute for Nuclear Astrophysics, the Kavli Institute for Particle Astrophysics and Cosmology, the Korean Scientist Group, the Chinese Academy of Sciences (LAMOST), Los Alamos National Laboratory, the Max-Planck-Institute for Astronomy (MPIA), the Max-Planck-Institute for Astrophysics (MPA), New Mexico State University, Ohio State University, University of Pittsburgh, University of Portsmouth, Princeton University, the United States Naval Observatory, and the University of Washington. 


\section{REFERENCES}

Adelman-McCarthy, J. K., et al. 2006, ApJS, 162, 38

Arav, N., et al. 2001, ApJ, 561, 118

Barlow, T. A., Junkkarinen, V. T., \& Burbidge, E. M. 1989, ApJ, 347, 674

Barlow, T. A., Junkkarinen, V. T., Burbidge, E. M., Weymann, R. J., Morris, S. L., \& Korista, K. T. 1992, ApJ, 397, 81

Barlow, T. A. 1994, PhD Thesis, University of California, San Diego.

Blanton, M. R., Lin, H., Lupton, R. H., Maley, F. M., Young, N., Zehavi, I., \& Loveday, J. 2003, AJ, 125, 2276

Boyle, B. J. 1990, MNRAS, 243, 231

Eisenstein, D. J. et al. 2001, AJ, 122, 2267

Elvis, M. 2000, ApJ, 545, 63

Foltz, C., Wilkes, B., Weymann, R., \& Turnshek, D. 1983, PASP, 95, 341

Foltz, C. B., Weymann, R. J., Morris, S. L., \& Turnshek, D. A. 1987, ApJ, 317, 450

Fukugita, M., Ichikawa, T., Gunn, J. E., Doi, M., Shimasaku, K., \& Schneider, D. P. 1996, AJ, 111, 1748

Ganguly, R., Charlton, J. C., \& Eracleous, M. 2001, ApJ, 556, L7

Green, P. J., Aldcroft, T. L., Mathur, S., Wilkes, B. J., \& Elvis, M. 2001, ApJ, 558, 109

Gunn, J. E. et al. 1998, AJ, 116, 3040

Gunn, J. E., et al. 2006, AJ, 131, 2332

Hall, P. B., et al. 2002, ApJS, 141, 267

Hamann, F., Barlow, T. A., Beaver, E. A., Burbidge, E. M., Cohen, R. D., Junkkarinen, V., \& Lyons, R. 1995, ApJ, 443, 606

Hamann, F., Barlow, T. A., \& Junkkarinen, V. 1997, ApJ, 478, 87

Hamann, F. 1998, ApJ, 500, 798 
Hamann, F., \& Sabra, B. 2004, ASP Conf. Ser. 311: AGN Physics with the Sloan Digital Sky Survey, 311, 203

Hewett, P. C., \& Foltz, C. B. 2003, AJ, 125, 1784

Hogg, D. W., Finkbeiner, D. P., Schlegel, D. J., \& Gunn, J. E. 2001, AJ, 122, 2129

Ivezić, Z., et al. 2004, AGN Physics with the Sloan Digital Sky Survey, Proceedings of a conference held in Princeton, NJ, USA, 27-31 July 2003, Edited by Gordon T. Richards and Patrick B. Hall, ASP Conference Series, Volume 311. San Francisco: Astronomical Society of the Pacific, 2004., p.437, 437

Laor, A., Bahcall, J. N., Jannuzi, B. T., Schneider, D. P., Green, R. F., \& Hartig, G. F. 1994, ApJ, 420, 110

Lupton, R., Gunn, J. E., Ivezić, Z., Knapp, G. R., Kent, S., \& Yasuda, N. 2001, in ASP Conf. Ser. 238, Astronomical Data Analysis Software and Systems X, ed. F. R. Harnden, Jr., F. A. Primini, and H. E. Payne (San Francisco: Astr. Soc. Pac.), p. 269, astro$\operatorname{ph}[0101420]$

Ma, F. 2002, MNRAS, 335, L99

Misawa, T., Eracleous, M., Charlton, J. C., \& Tajitsu, A. 2005, ApJ, 629, 115

Murray, N., Chiang, J., Grossman, S. A., \& Voit, G. M. 1995, ApJ, 451, 498

Narayanan, D., Hamann, F., Barlow, T., Burbidge, E. M., Cohen, R. D., Junkkarinen, V., \& Lyons, R. 2004, ApJ, 601, 715

Pier, J. R., et al. 2003, AJ, 125, 1559

Proga, D., Stone, J. M., \& Kallman, T. R. 2000, ApJ, 543, 686

Reichard, T. A., et al. 2003, AJ, 126, 2594

Richards, G. T., Vanden Berk, D. E., Reichard, T. A., Hall, P. B., Schneider, D. P., SubbaRao, M., Thakar, A. R., \& York, D. G. 2002, AJ, 124, 1

Richards, G. T. et al. 2002, AJ, 123, 2945

Rupke, D. S., Veilleux, S., \& Sanders, D. B. 2002, ApJ, 570, 588

Smith, J. A. et al. 2002, AJ, 123, 2121

Smith, L. J., \& Penston, M. V. 1988, MNRAS, 235, 551 
Stoughton, C., et al. 2002, AJ, 123, 485

Strauss, M. A. et al. 2002, AJ, 124, 1810

Trump, J.R., et al. 2006 ApJ, Submitted.

Turnshek, D. A., Grillmair, C. J., Foltz, C. B., \& Weymann, R. J. 1988, ApJ, 325, 651

Vanden Berk, D. E., et al. 2001, AJ, 122, 549

Vanden Berk, D. E., et al. 2004, ApJ, 601, 692

Vilkoviskij, E. Y., \& Irwin, M. J. 2001, MNRAS, 321, 4

Weymann, R. J., Morris, S. L., Foltz, C. B., \& Hewett, P. C. 1991, ApJ, 373, 23

Wilhite, B. C., Vanden Berk, D. E., Kron, R. G., Schneider, D. P., Pereyra, N., Brunner, R. J., Richards, G. T., \& Brinkmann, J. V. 2005, ApJ, 633, 638

Wilhite, B. C., Vanden Berk, D. E., Brunner, R. J., \& Brinkmann, J. V. 2006, ApJ, 641, 78

Wilkes, B. J. 1984, MNRAS, 207, 73

Wills, B. J., Brotherton, M. S., Fang, D., Steidel, C. C., \& Sargent, W. L. W. 1993, ApJ, 415,563

Wise, J. H., Eracleous, M., Charlton, J. C., \& Ganguly, R. 2004, ApJ, 613, 129

York, D. G., et al. 2000, AJ, 120, 1579

Yuan, M. J., \& Wills, B. J. 2003, ApJ, 593, L11 


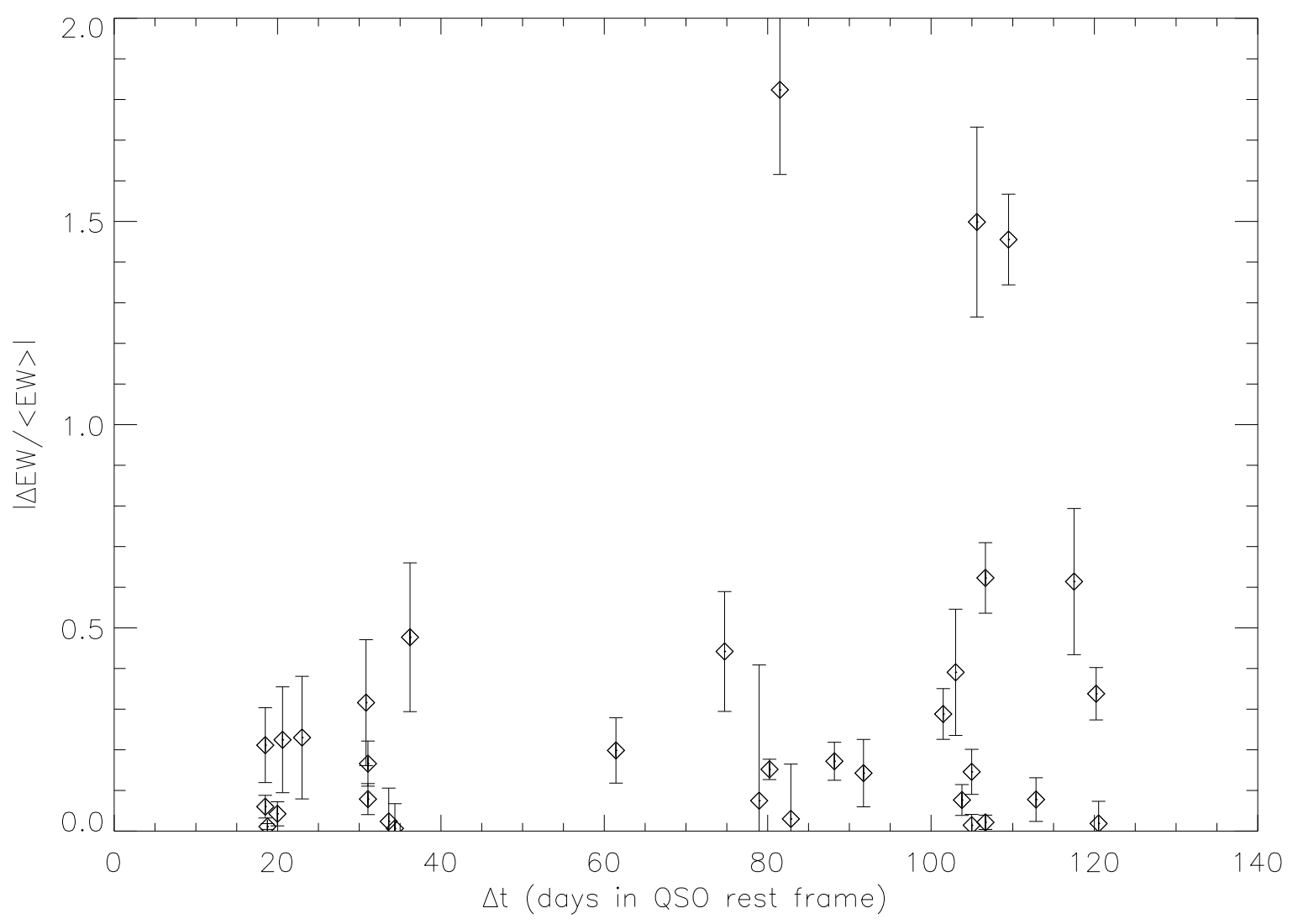

Fig. 1.- Absolute fractional change in BAL equivalent width as a function of epoch separation time, measured in days in the quasar rest frame. Equivalent width variability is strongest for BALs observed over long timescales, indicating the observation of real variability. 


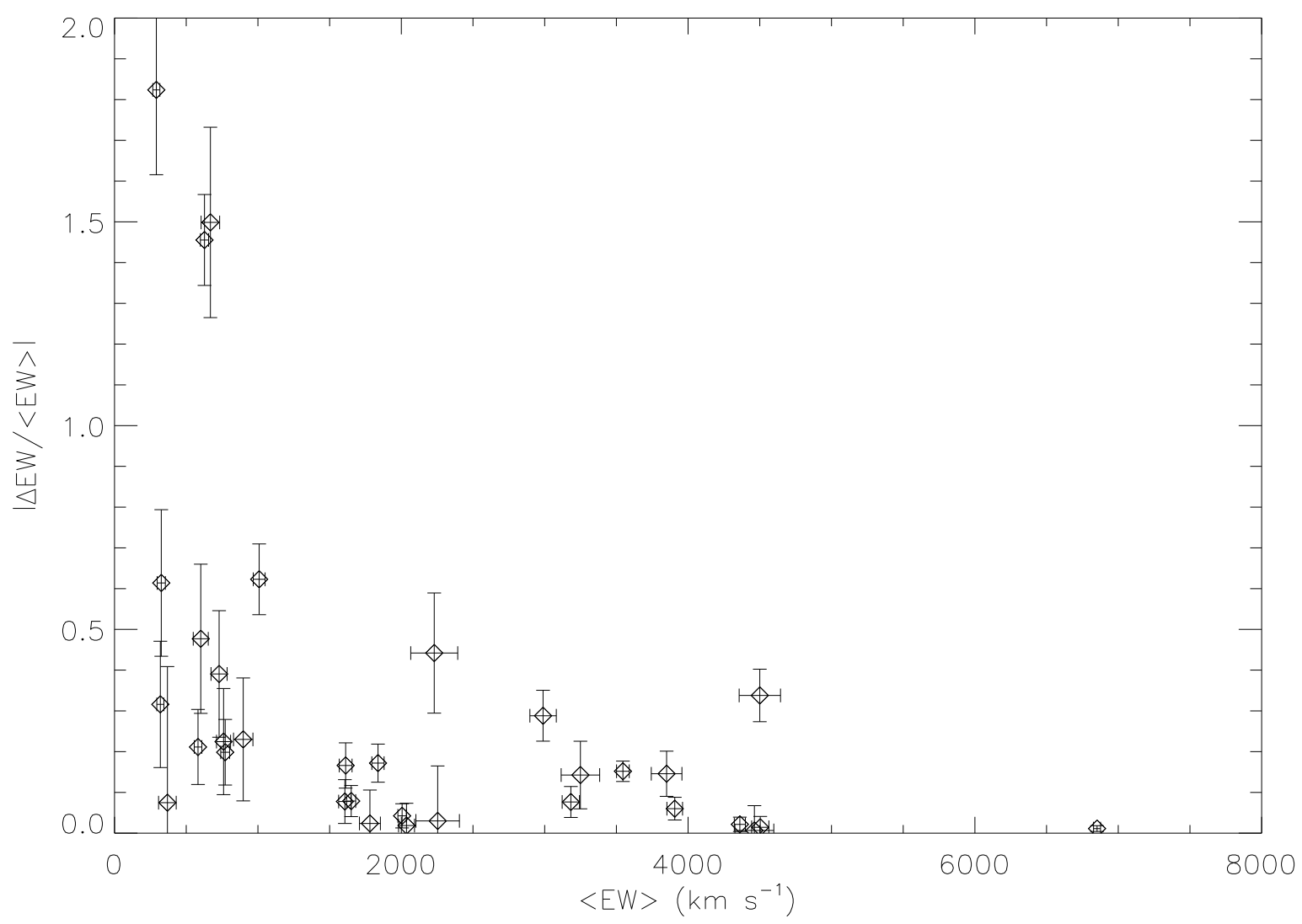

Fig. 2.- Absolute fractional change in BAL equivalent width as a function of the average equivalent width. The greatest variability occurs in the features with relatively small equivalent widths, in accordance with the findings of Barlow (1994). 


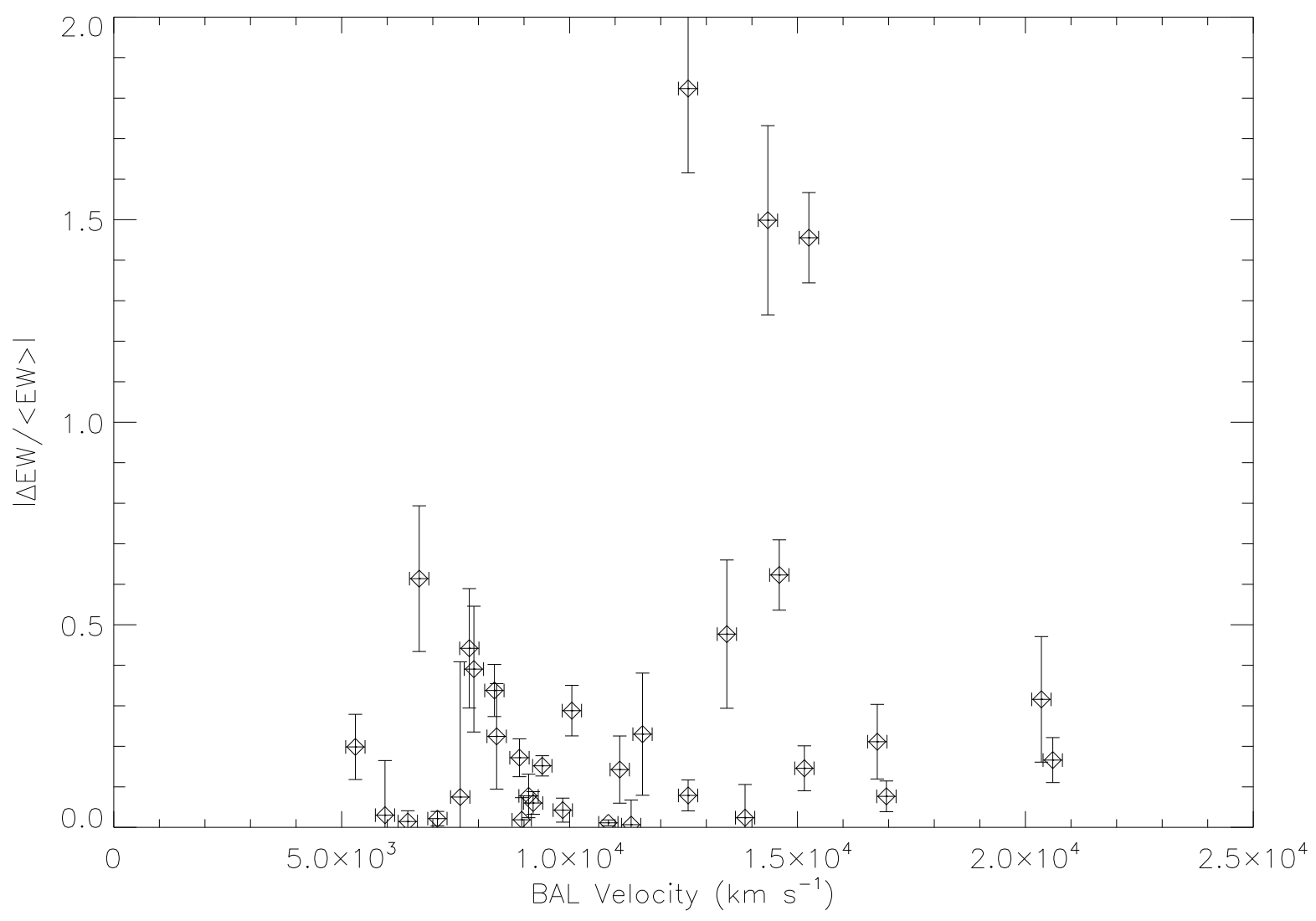

Fig. 3.- Absolute fractional change in BAL equivalent width as a function of BAL velocity. The three most variable features are found at velocities exceeding $12,000 \mathrm{~km} \mathrm{~s}^{-1}$, a region of predicted instability in the Proga et al. (2000) disk wind model. 


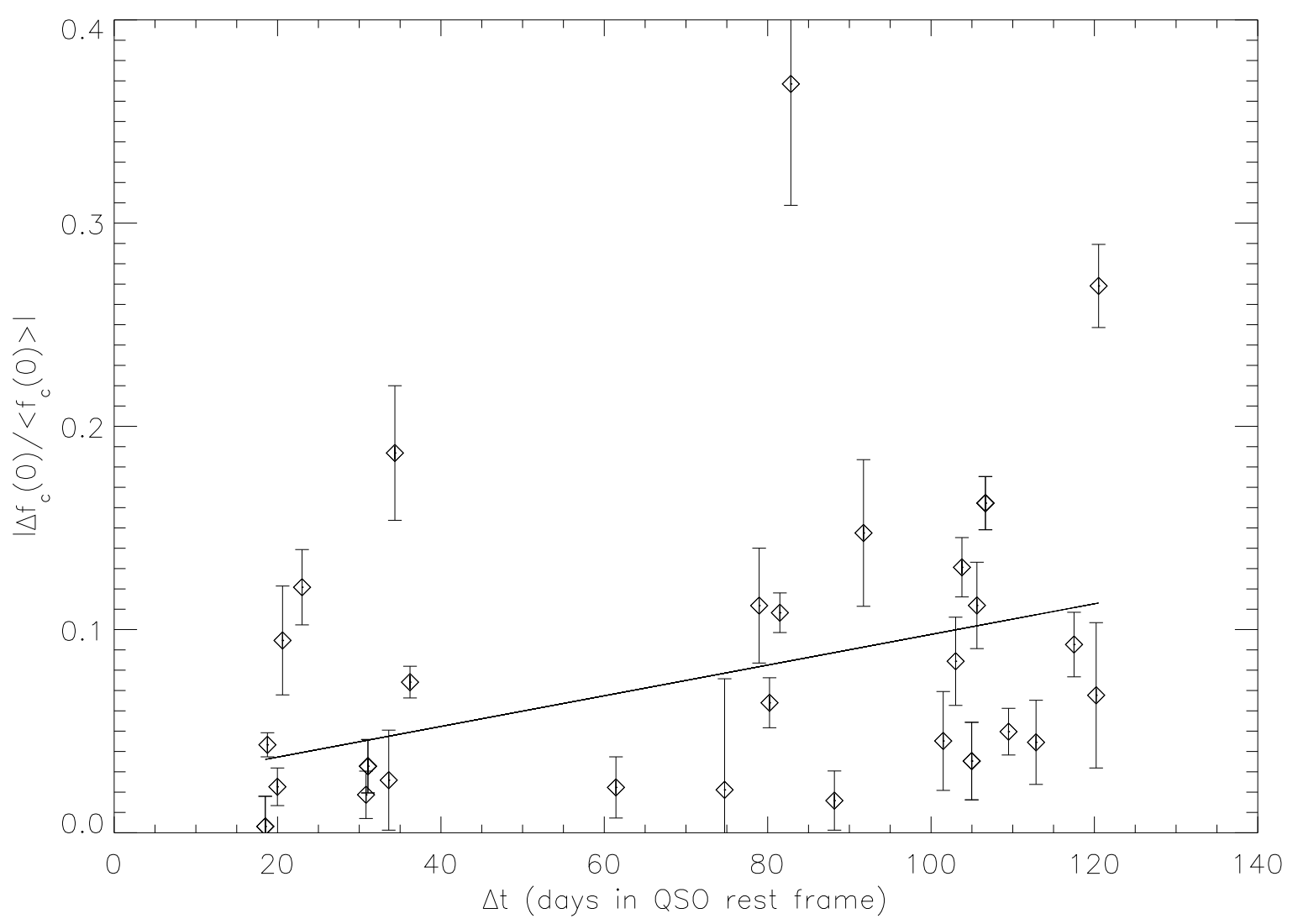

Fig. 4.- Absolute fractional change in the fitted continuum flux at $1549 \AA$ as a function of epoch separation time, measured in days in the quasar rest frame. A linear weighted least squares fit is overplotted. The Spearman Rank Correlation of these data returns a positive correlation with a significance of 0.0098 , confirming that real continuum variability occurs in our BALQSO sample. 


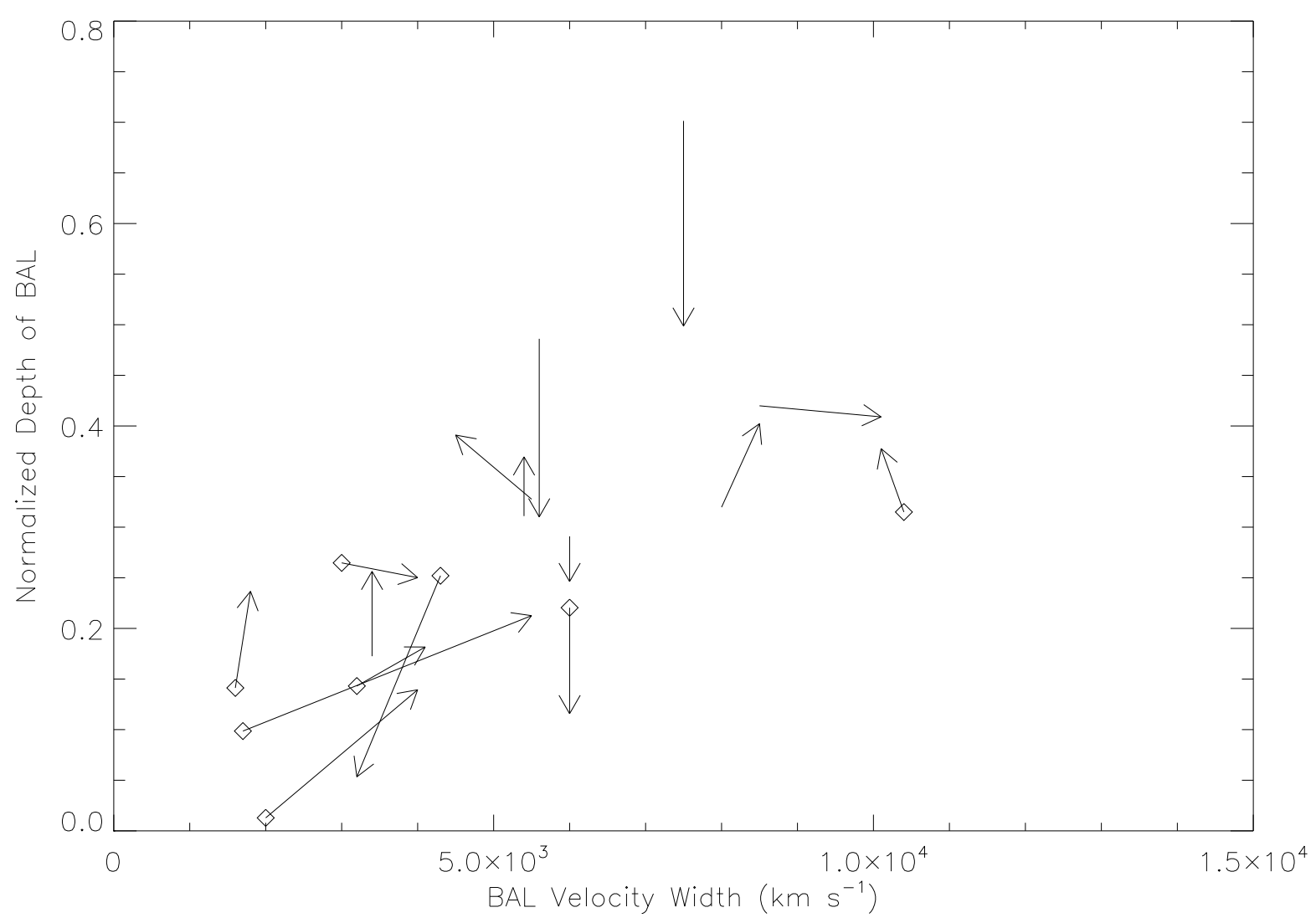

Fig. 5.- Direction and magnitude of changes in depth (in units of normalized flux density) versus velocity width for the 16 significantly variable BALs. The variable BALs that are associated with the 15 quasars found to have significantly variable underlying continua are marked with diamonds at the first epoch measurements. 


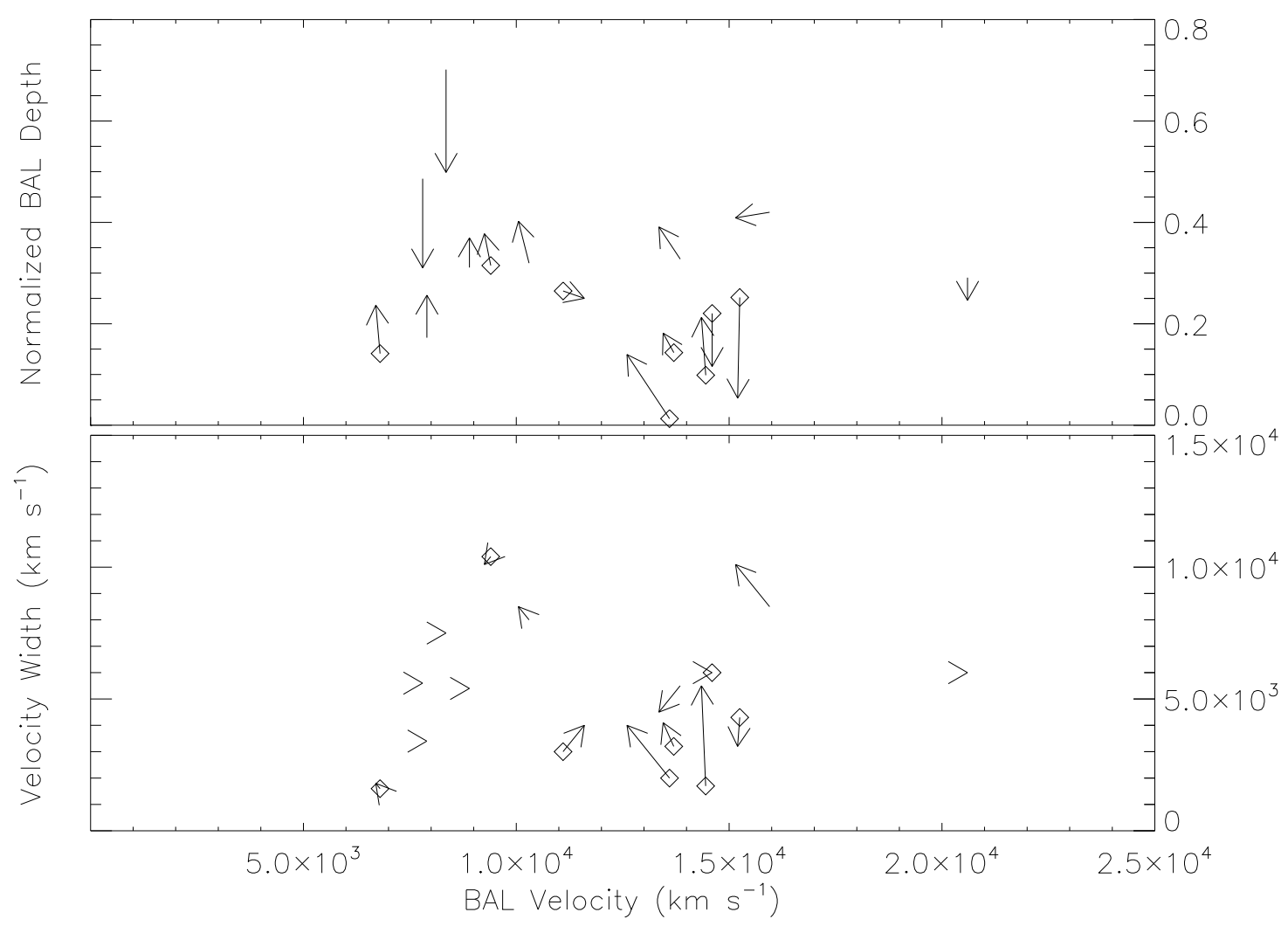

Fig. 6.- Top Panel: Direction and magnitude of change in depth (in units of normalized flux density) versus velocity for the 16 most significantly variable BALs. Bottom Panel: Direction and magnitude of changes in velocity width versus velocity for the same BALs. The variable BALs that are associated with the 15 quasars found to have significantly variable underlying continua are marked with diamonds at the first epoch measurements. 
Table 1. SDSS Spectral Observations

\begin{tabular}{|c|c|c|c|c|}
\hline Object $^{a}$ & SDSS J & $\mathrm{Z}_{h s n}{ }^{b}$ & $\mathrm{MJD}_{h s n}$ & $\mathrm{MJD}_{l s n}$ \\
\hline 1 & $115031.03-004403.1$ & 2.3921 & 51943 & 51662 \\
\hline 2 & $130058.13+010551.5$ & 1.9015 & 51689 & 51994 \\
\hline 3 & $132304.58-003856.5$ & 1.8267 & 51984 & 51665 \\
\hline 4 & $131853.45+002211.5$ & 2.0737 & 51984 & 51665 \\
\hline 5 & $132742.92+003532.6$ & 1.8736 & 51959 & 51663 \\
\hline 6 & $133150.51+004518.7$ & 1.8869 & 51955 & 51662 \\
\hline 7 & $134544.54+002810.7$ & 2.4528 & 51943 & 51666 \\
\hline 8 & $143641.24+001558.9$ & 1.8659 & 51637 & 51690 \\
\hline 9 & $144959.96+003225.3$ & 1.7217 & 51994 & 51666 \\
\hline 10 & $150206.66-003606.9$ & 2.1998 & 51990 & 51614 \\
\hline 11 & $150109.13-011502.7$ & 2.1278 & 51990 & 51614 \\
\hline 12 & $170633.06+615715.0$ & 2.0115 & 51695 & 51780 \\
\hline 13 & $172001.31+621245.7$ & 1.7642 & 51694 & 51789 \\
\hline 14 & $234506.31+010135.5$ & 1.7969 & 51877 & 51783 \\
\hline 15 & $031828.90-001523.1$ & 1.9820 & 51929 & 51821 \\
\hline 16 & $004527.68+143816.1$ & 1.9842 & 51868 & 51812 \\
\hline 17 & $093620.52+004649.2$ & 1.7175 & 52314 & 52027 \\
\hline 18 & $131305.74+015926.9$ & 2.0169 & 52295 & 52029 \\
\hline 19 & $081822.63+434633.8$ & 2.0430 & 52207 & 51959 \\
\hline 20 & $081416.75+435405.0$ & 1.7036 & 52207 & 51959 \\
\hline 21 & $153703.94+533219.9$ & 2.4035 & 52374 & 52442 \\
\hline 22 & $224019.01+144435.5$ & 2.2424 & 52520 & 52264 \\
\hline 23 & $143130.04+570139.0$ & 1.8006 & 52346 & 52433 \\
\hline 24 & $144403.97+565751.3$ & 1.8544 & 52347 & 52435 \\
\hline 25 & $145428.52+571441.3$ & 3.2583 & 52347 & 52435 \\
\hline 26 & $160649.24+451051.6$ & 2.8256 & 52443 & 52355 \\
\hline 27 & $102250.16+483631.1$ & 2.0658 & 52347 & 52674 \\
\hline 28 & $075010.17+304032.3$ & 1.8954 & 52346 & 52663 \\
\hline 29 & $081657.55+060441.7$ & 2.0111 & 52962 & 52737 \\
\hline
\end{tabular}


Note. — "hsn" and "lsn" refer to high- and low-S/N epoch observations respectively.

aThese object numbers are used for internal reference and correspond to the labeling of individual quasars in Tables $2 \&$ 3, and Figure A1.

${ }^{\mathrm{b}}$ The quasar redshift from the SDSS for the high-S/N epoch observation. 
Table 2. Continuum Fitting Parameters (in quasar rest frame)

\begin{tabular}{|c|c|c|c|c|c|c|}
\hline Object $^{a}$ & $\lambda_{b 1}(\AA)$ & $\lambda_{b 2}(\AA)$ & $m_{1}\left(10^{-5}\right)$ & $m_{2}\left(10^{-5}\right)$ & $f_{c}(0)_{1}$ & $f_{c}(0)_{2}$ \\
\hline 1 & 1440 & 1460 & $-0.95 \pm 0.31$ & $-0.33 \pm 0.22$ & $1.27 \pm 0.07$ & $1.84 \pm 0.05$ \\
\hline 2 & 1430 & 1450 & $-0.39 \pm 0.31$ & $-0.25 \pm 0.38$ & $6.68 \pm 0.08$ & $6.45 \pm 0.10$ \\
\hline 3 & 1440 & 1460 & $-4.40 \pm 0.80$ & $-3.55 \pm 0.52$ & $11.42 \pm 0.21$ & $11.93 \pm 0.13$ \\
\hline 4 & 1430 & 1445 & $-0.50 \pm 0.39$ & $-0.82 \pm 0.32$ & $9.11 \pm 0.10$ & $8.00 \pm 0.08$ \\
\hline 5 & 1440 & 1460 & $-0.86 \pm 0.53$ & $-2.12 \pm 0.41$ & $7.35 \pm 0.13$ & $8.00 \pm 0.10$ \\
\hline 6 & 1440 & 1460 & $-1.87 \pm 0.43$ & $-1.57 \pm 0.44$ & $6.44 \pm 0.11$ & $6.15 \pm 0.11$ \\
\hline 7 & 1440 & 1460 & $-3.64 \pm 0.32$ & $-2.90 \pm 0.31$ & $8.77 \pm 0.07$ & $8.22 \pm 0.07$ \\
\hline 8 & 1440 & 1460 & $0.82 \pm 0.32$ & $-0.70 \pm 0.39$ & $8.39 \pm 0.08$ & $8.42 \pm 0.10$ \\
\hline 9 & 1440 & 1460 & $-4.25 \pm 0.54$ & $-5.48 \pm 0.42$ & $7.45 \pm 0.14$ & $9.77 \pm 0.11$ \\
\hline 10 & 1440 & 1460 & $-2.25 \pm 0.38$ & $-4.18 \pm 0.27$ & $6.91 \pm 0.09$ & $7.59 \pm 0.07$ \\
\hline 11 & 1440 & 1460 & $0.37 \pm 0.47$ & $-0.44 \pm 0.26$ & $3.53 \pm 0.12$ & $3.78 \pm 0.06$ \\
\hline 12 & 1440 & 1460 & $-3.21 \pm 0.42$ & $-2.23 \pm 0.35$ & $9.13 \pm 0.11$ & $8.92 \pm 0.09$ \\
\hline 13 & 1440 & 1460 & $0.31 \pm 0.53$ & $-0.84 \pm 0.54$ & $5.28 \pm 0.14$ & $6.37 \pm 0.14$ \\
\hline 14 & 1440 & 1460 & $-2.02 \pm 0.38$ & $-2.28 \pm 0.38$ & $5.67 \pm 0.10$ & $5.53 \pm 0.10$ \\
\hline 15 & 1440 & 1460 & $-1.45 \pm 0.36$ & $-0.10 \pm 0.33$ & $16.10 \pm 0.09$ & $14.95 \pm 0.08$ \\
\hline 16 & 1440 & 1460 & $-16.49 \pm 0.51$ & $-11.70 \pm 0.48$ & $30.24 \pm 0.13$ & $28.94 \pm 0.12$ \\
\hline 17 & 1440 & 1460 & $-2.59 \pm 0.67$ & $-3.88 \pm 0.53$ & $10.74 \pm 0.17$ & $9.61 \pm 0.14$ \\
\hline 18 & 1440 & 1460 & $-2.94 \pm 0.44$ & $-2.61 \pm 0.25$ & $8.68 \pm 0.11$ & $8.54 \pm 0.06$ \\
\hline 19 & 1440 & 1460 & $-4.03 \pm 0.42$ & $-2.55 \pm 0.26$ & $13.24 \pm 0.11$ & $11.87 \pm 0.06$ \\
\hline 20 & 1450 & 1460 & $1.22 \pm 0.49$ & $0.38 \pm 0.30$ & $3.92 \pm 0.11$ & $3.39 \pm 0.07$ \\
\hline 21 & 1440 & 1460 & $-5.17 \pm 0.29$ & $-4.91 \pm 0.41$ & $13.02 \pm 0.07$ & $13.31 \pm 0.10$ \\
\hline 22 & 1440 & 1460 & $-1.21 \pm 0.75$ & $-0.30 \pm 0.28$ & $6.54 \pm 0.18$ & $7.31 \pm 0.07$ \\
\hline 23 & 1500 & 1520 & $-9.83 \pm 0.87$ & $-3.37 \pm 1.02$ & $23.76 \pm 0.20$ & $22.95 \pm 0.23$ \\
\hline 24 & 1455 & 1470 & $-6.26 \pm 0.60$ & $-6.30 \pm 0.60$ & $17.18 \pm 0.14$ & $16.86 \pm 0.14$ \\
\hline 25 & 1440 & 1460 & $-0.81 \pm 0.16$ & $-0.81 \pm 0.15$ & $2.08 \pm 0.04$ & $1.89 \pm 0.03$ \\
\hline 26 & 1440 & 1460 & $1.50 \pm 0.41$ & $1.86 \pm 0.23$ & $5.89 \pm 0.10$ & $6.65 \pm 0.06$ \\
\hline 27 & 1440 & 1460 & $-1.55 \pm 0.36$ & $-3.89 \pm 0.38$ & $9.08 \pm 0.09$ & $10.70 \pm 0.09$ \\
\hline 28 & 1450 & 1460 & $-3.32 \pm 0.42$ & $-3.69 \pm 0.41$ & $11.73 \pm 0.09$ & $11.16 \pm 0.09$ \\
\hline 29 & 1440 & 1460 & $-1.36 \pm 1.27$ & $-1.21 \pm 0.37$ & $6.17 \pm 0.32$ & $6.04 \pm 0.09$ \\
\hline
\end{tabular}


Note. - Quasar rest-frame wavelengths presented in columns 2 and 3 indicate the spectral ranges used for the blue side of each linear continuum fit. The spectral ranges for the red side are fixed at $1680-1700 \AA$ for each spectrum and are therefore omitted from the table (see $\S 3.1$ for discussion). The slopes (columns 4 and 5) and flux density values at $v=0 \mathrm{~km} \mathrm{~s}^{-1}$ (columns 6 and 7), which are returned by a linear fit for each epoch, are shown. Subscripts 1 and 2 refer to the first and second epochs of observation respectively.

${ }^{a}$ Object names corresponding to these numbers can be found in Table 1. 
Table 3. Two-Epoch BAL Measurements $\left(\mathrm{km} \mathrm{s}^{-1}\right.$ in quasar rest frame)

\begin{tabular}{lcccccc}
\hline \hline Object $^{*}$ & $\Delta t_{\text {qso }}($ days $)$ & $v_{\text {max }}$ & $v_{\text {min }}$ & $E W_{1}$ & $E W_{2}$ & $\Delta E W /<E W>$ \\
\hline 1 & 83 & -7600 & -4300 & $2220 \pm 261$ & $2287 \pm 155$ & $+0.030 \pm 0.135$ \\
$2 \mathrm{a}^{\dagger}$ & 105 & -20200 & -10100 & $3570 \pm 122$ & $4131 \pm 175$ & $+0.146 \pm 0.055$ \\
$2 \mathrm{~b}$ & 105 & -9300 & -3600 & $4536 \pm 71$ & $4471 \pm 97$ & $-0.014 \pm 0.027$ \\
3 & 113 & -10800 & -7400 & $1669 \pm 74$ & $1544 \pm 45$ & $-0.078 \pm 0.054$ \\
4 & 104 & -21300 & -12600 & $3061 \pm 91$ & $3304 \pm 79$ & $+0.076 \pm 0.038$ \\
$5^{\dagger}$ & 103 & -9600 & -6200 & $587 \pm 95$ & $872 \pm 60$ & $+0.391 \pm 0.155$ \\
$6^{\dagger}$ & 101 & -14300 & -5800 & $2558 \pm 125$ & $3419 \pm 135$ & $+0.288 \pm 0.062$ \\
$7^{\dagger}$ & 80 & -14600 & -4200 & $3276 \pm 60$ & $3814 \pm 65$ & $+0.152 \pm 0.025$ \\
$8 \mathrm{a}$ & 18 & -17600 & -15900 & $644 \pm 34$ & $521 \pm 41$ & $-0.211 \pm 0.092$ \\
$8 \mathrm{~b}$ & 18 & -13100 & -5300 & $3790 \pm 68$ & $4024 \pm 85$ & $+0.060 \pm 0.028$ \\
9 & 121 & -11600 & -6300 & $2056 \pm 95$ & $2017 \pm 57$ & $-0.019 \pm 0.055$ \\
$10^{\dagger}$ & 118 & -7600 & -5800 & $226 \pm 49$ & $426 \pm 29$ & $+0.614 \pm 0.180$ \\
$11^{\dagger}$ & 120 & -12100 & -4600 & $5261 \pm 259$ & $3740 \pm 128$ & $-0.338 \pm 0.064$ \\
12 & 61 & -6900 & -3700 & $695 \pm 46$ & $848 \pm 41$ & $+0.199 \pm 0.080$ \\
13 & 34 & -15100 & -7600 & $4477 \pm 226$ & $4448 \pm 150$ & $-0.007 \pm 0.061$ \\
$14^{\dagger}$ & 34 & -16600 & -11100 & $1802 \pm 103$ & $1760 \pm 103$ & $-0.024 \pm 0.082$ \\
$15^{\dagger}$ & 36 & -15500 & -11400 & $458 \pm 31$ & $745 \pm 100$ & $+0.477 \pm 0.183$ \\
16 & 19 & -18100 & -3600 & $6890 \pm 35$ & $6814 \pm 34$ & $-0.011 \pm 0.007$ \\
$17^{\dagger}$ & 106 & -17100 & -11600 & $168 \pm 99$ & $1169 \pm 83$ & $+1.499 \pm 0.234$ \\
$18^{\dagger}$ & 88 & -11600 & -6200 & $1679 \pm 76$ & $1995 \pm 40$ & $+0.172 \pm 0.047$ \\
$19^{\dagger}$ & 81 & -14600 & -10600 & $26 \pm 41$ & $558 \pm 27$ & $+1.824 \pm 0.208$ \\
20 & 92 & -15100 & -7100 & $3018 \pm 207$ & $3481 \pm 171$ & $+0.143 \pm 0.083$ \\
21 & 20 & -13600 & -6100 & $1963 \pm 34$ & $2048 \pm 48$ & $+0.042 \pm 0.030$ \\
22 & 79 & -8600 & -6600 & $383 \pm 111$ & $355 \pm 53$ & $-0.075 \pm 0.334$ \\
$23 \mathrm{a}^{\dagger}$ & 31 & -23600 & -17600 & $1746 \pm 55$ & $1478 \pm 70$ & $-0.166 \pm 0.056$ \\
$23 \mathrm{~b}$ & 31 & -14800 & -10400 & $1715 \pm 39$ & $1585 \pm 50$ & $-0.079 \pm 0.038$ \\
24 & 31 & -21600 & -19100 & $269 \pm 34$ & $370 \pm 35$ & $+0.316 \pm 0.155$ \\
25 & 21 & -9600 & -7200 & $675 \pm 67$ & $846 \pm 72$ & $+0.225 \pm 0.130$ \\
$26^{\dagger}$ & 23 & -13600 & -9600 & $794 \pm 124$ & $1001 \pm 53$ & $+0.230 \pm 0.151$ \\
$27 \mathrm{a}^{\dagger}$ & 107 & -17600 & -11600 & $1323 \pm 63$ & $695 \pm 54$ & $-0.623 \pm 0.087$ \\
$27 \mathrm{~b}$ & 107 & -10600 & -3600 & $4314 \pm 60$ & $4408 \pm 49$ & $+0.022 \pm 0.018$
\end{tabular}


Table 3-Continued

\begin{tabular}{lcccccc}
\hline \hline Object* $^{*}$ & $\Delta t_{\text {qso }}($ days $)$ & $v_{\max }$ & $v_{\min }$ & $E W_{1}$ & $E W_{2}$ & $\Delta E W /<E W>$ \\
\hline $28^{\dagger}$ & 109 & -17400 & -13100 & $1084 \pm 39$ & $171 \pm 41$ & $-1.455 \pm 0.111$ \\
$29^{\dagger}$ & 75 & -10600 & -5000 & $2722 \pm 314$ & $1737 \pm 93$ & $-0.442 \pm 0.147$ \\
\hline
\end{tabular}

Note. - Columns 3 and 4 present the velocity boundaries of the BAL, shown as vertical dotted lines in Figure A1. Equivalent widths and fractional change in equivalent width are given in columns $5,6 \& 7$. Subscripts 1 and 2 are used to denote the first and second epoch observations respectively. Object 20 has separate upper and lower error bars, since measurements of fractional change as defined here cannot exceed 2.

*Object names corresponding to these numbers can be found in Table 1. For cases where multiple high-velocity BALs occur in a single quasar spectrum, letters (a \& b) are added to the object number to differentiate between the separate features.

${ }^{\dagger}$ Denotes significantly variable BALs, according to the criteria outlined in $§ 3.3$. 


\section{A. Spectral Observations}

Fig. A1.- Continuum-normalized two-epoch SDSS spectral observations for all sources analyzed in this paper. The top window presents the high signal-to-noise spectra in black, with the low signal-to-noise in gray, each with a 3-pixel smoothing. The center window shows the unsmoothed error spectrum for each epoch, and the lowest window displays the difference spectrum of the two epochs, with a 3-pixel smoothing. Vertical dotted lines overplot boundaries of BALs chosen for analysis. Numbers in the upper-left corner of each spectrum correspond to the object numbers used in Tables 1-3. Full object names for each quasar are given in Table 1.
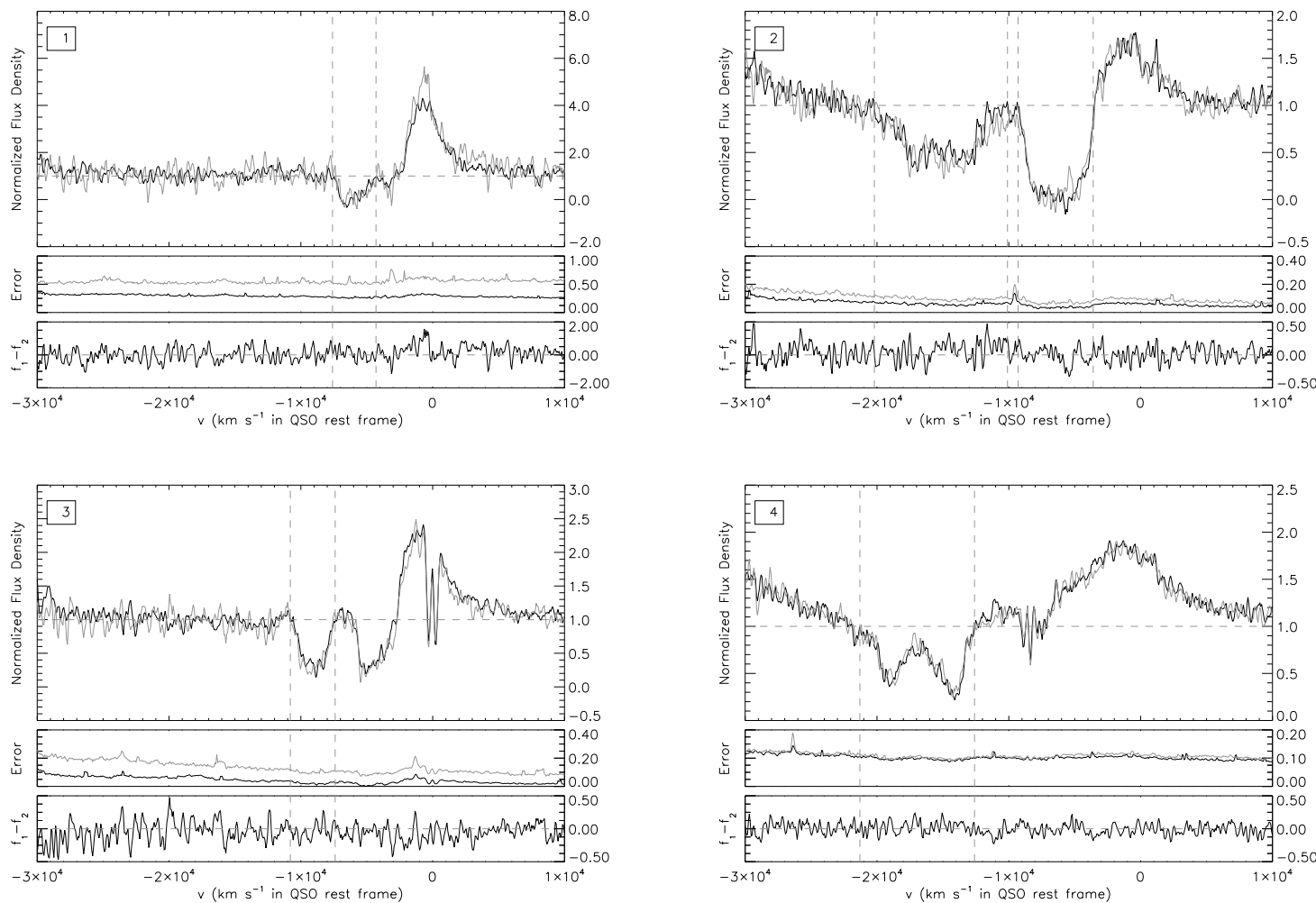

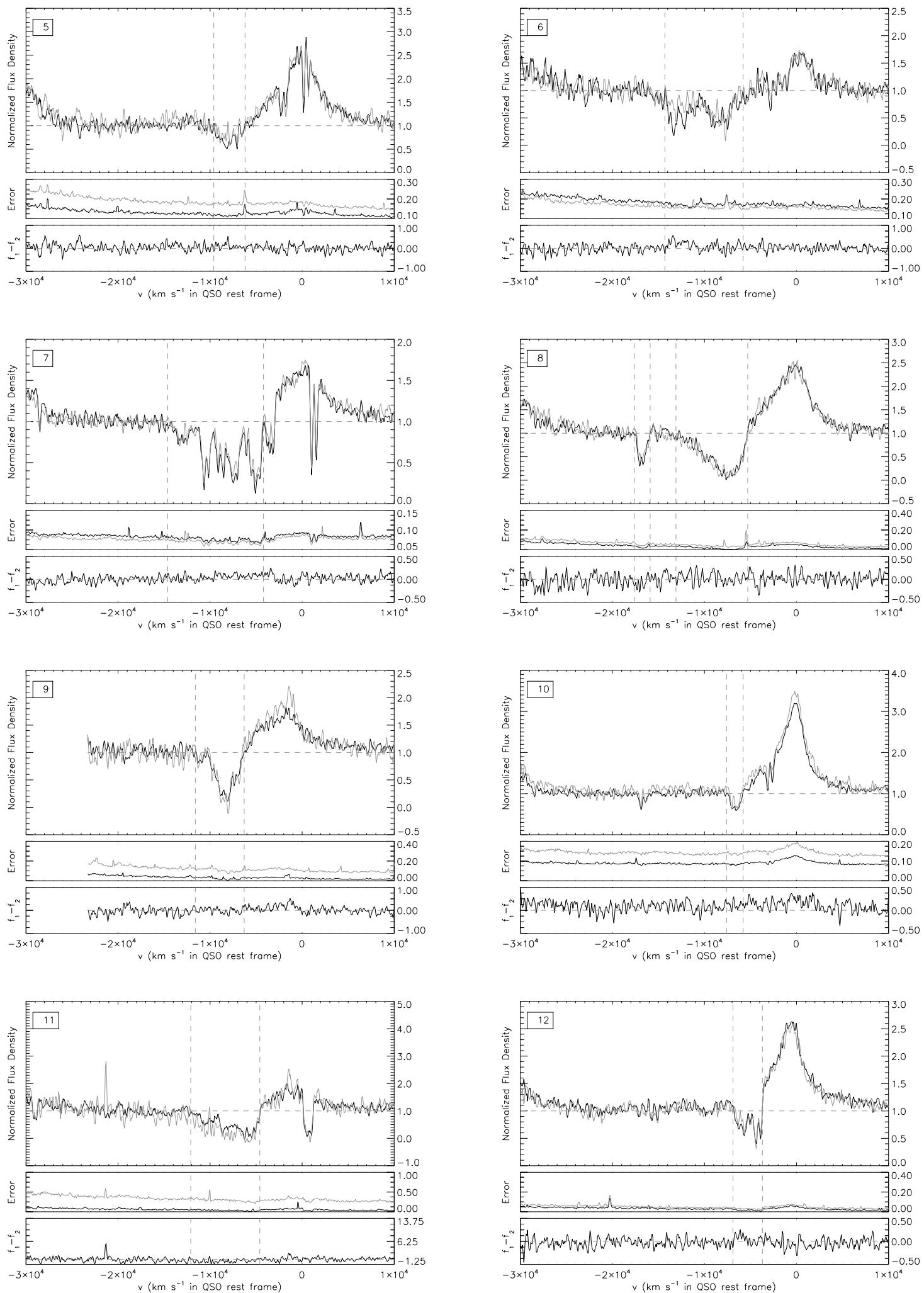

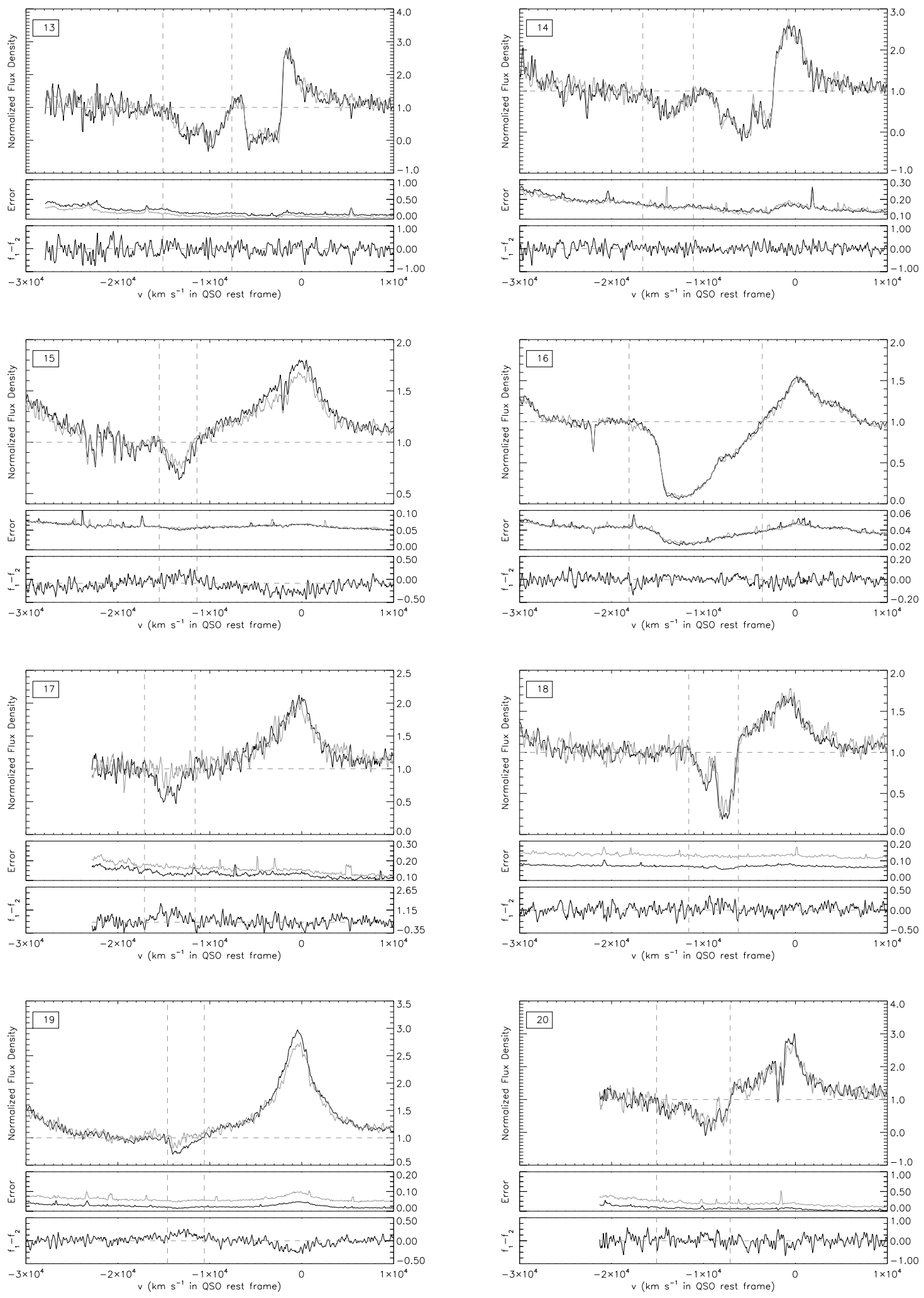

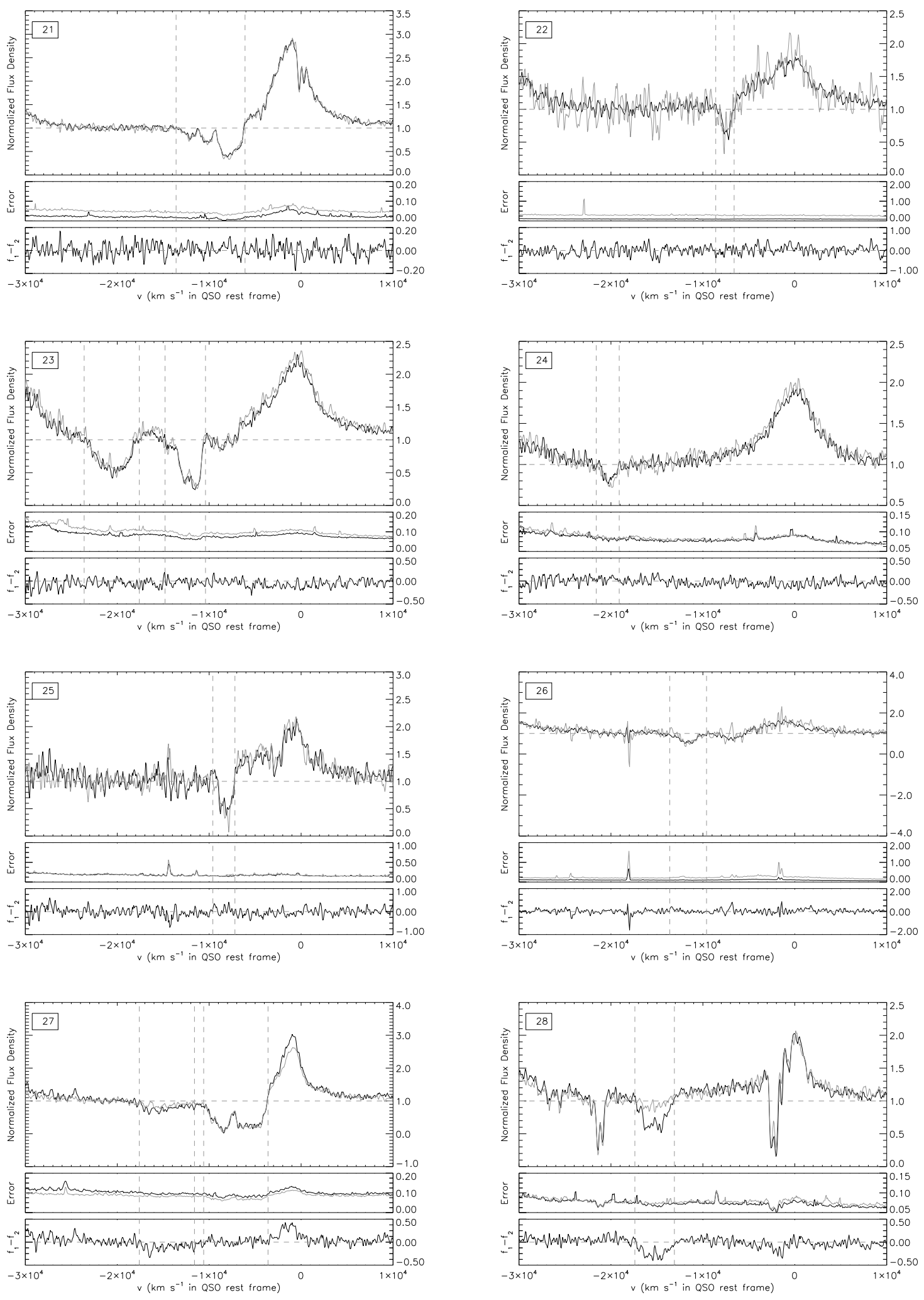


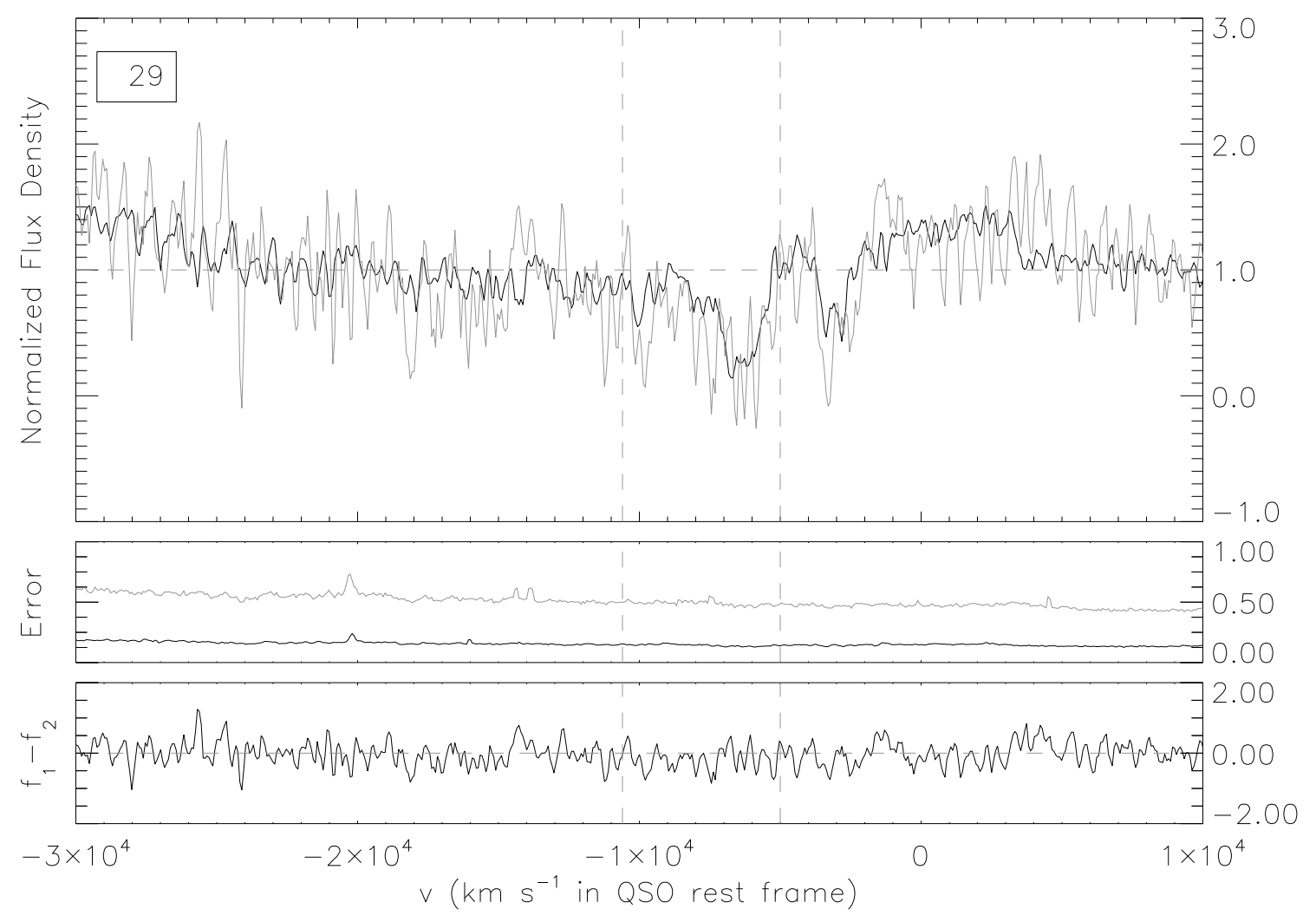

